\title{
Catálogo de la colección de cultivos de especies de hongos patógenos y simbiontes de insectos y otros artrópodos de la Argentina
}

\section{Catalogue of the culture collection of symbionts and pathogenic fungal species of insects and other arthropods from Argentina}

\section{Claudia C. López Lastra ${ }^{1 *}$, Romina G. Manfrino ${ }^{1}$, Mónica B. Rodríguez $^{2}$, Alejandra C. Gutierrez $^{1}$, Eliana Ordoqui ${ }^{1}$ \& Graciela T. Navone ${ }^{1,2}$}

${ }^{1}$ CEPAVE (CONICET-UNLP), La Plata, Buenos Aires, Argentina.

${ }^{2}$ FCNyM-UNLP, La Plata, Buenos Aires, Argentina.

*E-mail: claudia@cepave.edu.ar

\section{RESUMEN}

El presente trabajo, tiene como objetivo dar a conocer los datos actualizados e información relevante de las cepas de especies fúngicas entomopatógenas preservadas en la colección de cultivos del CEPAVE (Centro de Estudios Parasitológicos y de Vectores) CONICET-UNLP, Argentina. Los datos que se informan en este trabajo son de aislamientos nativos de diferentes regiones fitogeográficas de la Argentina. Para cada cepa se especifica: acrónimo, sustrato, especie de insecto hospedante, fecha de recolección, referencias de coordenadas geográficas y medio de cultivo en el que fue aislado. Los fines de esta Colección son de investigación, docencia y vinculación tecnológica a empresas. Su inicio fue en 1988 y, actualmente se encuentra registrada en la FELACC (Federación Latinoamericana de Colecciones de Cultivos) como Socio Institucional: № SI-06 y en la Federación Mundial de Colecciones de Cultivos (World Federation for Culture Collections - WFCC) (http://www.wfcc.info/). Siguiendo las pautas de organización de la WFCC esta colección está incorporada a: World Data Centre for Microorganisms (WDCM, № 973 CEP). Los métodos de preservación que se utilizan son: agua destilada estéril, papel, aceite mineral, sílica gel, freezer $-20{ }^{\circ} \mathrm{C}$ y $-70{ }^{\circ} \mathrm{C}$ y liofilización, siendo realizados rigurosos controles de calidad y de viabilidad de las cepas. Las cepas son caracterizadas por técnicas morfológicas y el $30 \%$ de los aislamientos fúngicos han sido caracterizados, además, mediante técnicas de biología molecular. En la actualidad, la colección mantiene más de 650 cepas correspondientes en su mayoría a los géneros Beauveria, Metarhizium, Isaria, Lecanicillium y algunas especies de Entomophthorales.

Palabras clave: aislamientos, Argentina, colección, hongos entomopatógenos, preservación.

\section{ABSTRACT}

This manuscript seeks to disclose the updated data and relevant information about the entomopathogenic fungi strains preserved at the Center for Parasitological and Vector Studies (CEPAVE) of the National Scientific and Technical Research Council and National University of La Plata (CONICET-UNLP), Argentina. The data provided here is the result of surveys of native isolates from the different phytogeographic regions of Argentina, specified as follows: acronym, substrate, host insect species, collection date, geographic coordinate references, and culture medium in which it was isolated; and the aim is to provide basic information about the organisms preserved in the afore mentioned microorganisms collection. This collection was developed for the purposes of research, teaching 
and technological associations with companies. It started in 1988, and it is registered in the Latin American Federation of Culture Collections (FELACC) as Institutional Partner: No. SI-06, and in the World Federation for Culture Collections (WFCC) (http://www.wfcc.info/). In accordance with the WFCC guidelines, this collection is incorporated as a partner to the World Data Centre for Microorganisms (WDCM, 973 CEP). The preservation methods used are sterile distilled water, paper, mineral oil, silica gel, freezer at -20 and $-70{ }^{\circ} \mathrm{C}$ and lyophilization, quality and viability controls are carried out. So far, $40 \%$ of the species have been identified by molecular techniques, and the study of the remaining isolated species is under development. The collection consists of more than 684 strains which belong mostly to the Beauveria, Metarhizium, Isaria, Lecanicillium genera, as well as some species of Entomophthorales.

Keywords: Argentina, collection, entomopathogenic fungi, isolates, preservation.

\section{INTRODUCCIÓN}

Las colecciones de cultivos Microbianos, son entidades en donde se realizan actividades cuyo objetivo es obtener, preservar, clasificar, estudiar y documentar de manera completa y accesible, cultivos de microorganismos axénicos de interés específico. La información generada a partir de las colecciones tiene relevancia en diferentes ámbitos como la salud, medicina, industria farmacéutica, agricultura y biotecnología, entre otras. La colección de cultivos microbianos del CEPAVE contribuye al conocimiento de la biodiversidad de los hongos patógenos de insectos, proporcionando recursos genéticos y favoreciendo la conservación del germoplasma para futuros estudios de investigación básicos y aplicados.

Existen pocos trabajos publicados sobre catálogos de colecciones de cultivos y muy pocos específicamente acerca de hongos entomopatógenos (Sosa-Gómez \& da Silva 2002; ARSEF Catálogo 2014; Montesinos-Matías et al. 2015) además de algunos otros catálogos de cultivos de hongos patógenos de plantas, de humanos o generales (Marcenaro Rodríguez 2013; Montes de Oca et al. 2008). Asimismo, han sido publicados de forma aislada datos sobre el manejo, organización y preservación de cultivos en colecciones (Fernández Andreu et al. 2005; Panizo et al. 2015).

La colección de hongos patógenos y simbiontes de insectos y de otros artrópodos del CEPAVE ha sido originada en 1988, inicialmente como resultado de los relevamientos y aislamientos realizados durante el período 1984-1988 que formaron parte del trabajo de tesis doctoral: "Incidencia de hongos patógenos de insectos de la Argentina" (FCNyMUNLP) (López Lastra, 1988). Posteriormente, se incluyeron aislamientos de cultivos fúngicos provenientes de relevamientos realizados en el marco de investigaciones y a partir de depósitos externos. Los cultivos puros axénicos fueron obtenidos a partir de insectos (principalmente de los Ordenes: Hemiptera, Hymenoptera, Lepidoptera, Diptera, Dermaptera, Coleoptera, Blattodea, Orthoptera y también a partir de Acari y de Araneae de la Clase Arachnida) recolectados en distintas localidades y regiones de la República Argentina. Existen actualmente 684 aislamientos fúngicos pertenecientes a 23 géneros diferentes. La mayor parte de la colección está compuesta por especies pertenecientes al Phylum Ascomycota, Phylum Zoopagomycota y Reino Straminipila (Gutiérrez et al. 2017). El propósito de este catálogo es contribuir al conocimiento de la diversidad de especies en cultivo y sus aislamientos preservados en la colección comparando, además, los métodos de preservación usados en su mantenimiento.

\section{MATERIAL Y MÉTODOS}

La identificación de las especies fue realizada siguiendo las claves taxonómicas citadas en (Humber 2012) y muchas especies fueron además confirmadas por este especialista. Los fines de la colección de hongos entomopatógenos de la cual se presenta el catálogo actual son de investigación, docencia y transferencia tecnológica. El relevamiento de las especies y cepas fue realizado por medio de la recolección de muestras de insectos y de otros artrópodos en distintos tipos de ambientes y regiones de la Argentina, así como a partir de muestras de suelo desde el año 1984 hasta el presente, siendo todos los aislamientos nativos. Los métodos de preservación en que se mantienen las cepas son: freezer $-20^{\circ} \mathrm{C}$ y freezer $-70^{\circ} \mathrm{C}$ en glicerol, agua destilada estéril, papel, arena, sílicagel y liofilización (López Lastra \& Gutierrez 2019).

Para cada cepa ingresada a la colección se ha confeccionado una ficha de ingreso o historial (Estrada \& Vélez 2003, Montesinos-Matías et al. 2015; Panizo et al. 
2015 Gutierrez et al. 2017) y fue organizado un sistema propio basado en el modelo de otras colecciones de cultivos específicas de entomopatógenos a nivel mundial, en el cual se incluyen los datos especificados a continuación: nombre de la especie fúngica, autor, acrónimo con número de cepa, Legit, del colector del material y entre paréntesis el nombre del especialista o curador que identificó el material. Luego se ingresan los datos del hospedador disponibles (Orden, Familia, Especie), datos de la recolección (fecha y datos de la localización geográfica: provincia, localidad, sitio), medio de cultivo en el que fue aislado y por último, se incluyen los datos de los métodos de preservación en que se conservó el material. En el catálogo los taxones se presentan por orden alfabético. La nomenclatura se corresponde con la ofrecida en Index Fungorum Partnership (2012) y 95 cepas del total del catálogo fueron secuenciadas a través del código de barras del proyecto fondo IBOL: (http://www.boldsystems. org/index.php/MAS_Management_RecordSearch). Ejemplo: Aspergillus brasiliensis Varga, Frisvad \& Samson ( $\mathrm{Ex}=$ A. niger)

CEP 491 P. Albornoz Medina (P. Albornoz Medina) Anastrepha fraterculus [Diptera: Tephritidae] 27 Mar. 09, ARG: Tucumán. Horco Molle, Yerba Buena [SDYA 1/4] [AD, $\left.\mathrm{F}-20^{\circ} \mathrm{C}\right]$

Estos datos se solicitan al ingreso y una vez verificada la pureza y viabilidad de los aislamientos, se le asigna a cada cepa un número de acceso definitivo que se ingresa a la base de datos. El catálogo se organizó por especie fúngica siguiendo el orden alfabético, empezando así por Alternaria sp., de acuerdo al criterio adoptado por el catálogo de ARSEF de EEUU. Se organizaron las abreviaturas en un listado a continuación.

\section{ABREVIATURAS}

Abr: abril

$A D$ : agua destilada estéril

Ago: agosto

AM: agar extracto de malta

APG: agar papa glucosa

ARG: Argentina

$A^{\circ}$ : arroyo

Cnia: Colonia

Dic: diciembre

Ene: enero

Est. Exp.: estación experimental

Feb: febrero

$\mathrm{F}-20^{\circ} \mathrm{C}$ : freezer $-20^{\circ} \mathrm{C}$

Jun: junio

Jul: julio

Leg. : legit
L: liofilización

Mar: marzo

May: mayo

Nac.: nacional

Nov: noviembre

$\mathrm{NI}$ : no identificado

Oct: octubre

P: papel

$\mathrm{PN}$ : parque nacional

PP: parque provincial

Pque.: parque

RP: reserva provincial

Set: septiembre

SDY: Sabouraud dextrosa agar

SEMA: Sabouraud maltosa agar

ACRÓNIMOS

ARSEF: Agricultural Research Service Entomopathogenic Fungal Culture Collection Ithaca, New York, EEUU.

CBS: Centralbureu Vor Schimmel Cultures, Wageningen, Holanda

CEP: CEPAVE (Centro de estudios Parasitológicos y de Vectores) La Plata, Argentina

LPSC: Instituto de Botánica Spegazzini, La Plata, Argentina

* Datos publicados

** La identificación fue confirmada por taxonomía molecular, con datos de secuenciamiento publicados.

\section{RESULTADOS}

Como resultado del relevamiento de la colección, en el siguiente listado se exponen las especies y la cantidad de aislamientos correspondientes a cada una, así como el número de cepas nativas para cada una.

\section{Listado de especies y cepas}

Alternaria sp.

CEP 430 J. Barneche (C.C. López Lastra) [Araneae] 1 Nov. 03 ARG: Buenos Aires, Magdalena, R.P. Pearson [SDYA 1/4] [AD]

Aspergillus sp.

CEP 284 G. Marti (G. Marti) Triatoma infestans [Heteroptera: Reduviidae] 15 Nov. 04 ARG: Mendoza., El Nochero [AM 2\%] [AD] (*)

CEP 279 G. Marti (G. Marti) Triatoma infestans [Heteroptera: Reduviidae] 15 Dic. 04 ARG: Mendoza. [AM 2\%] [AD] (*)

Aspergillus brasiliensis Varga, Frisvad \& Samson ( $\mathrm{Ex}=$ A. niger) CEP 491 P. Albornoz Medina (P. Albornoz Medina) Anastrepha fraterculus [Diptera: Tephritidae] 27 Mar. 09, ARG: Tucumán. 
Horco Molle, Yerba Buena [SDYA 1/4] [AD, F-20 2 C]

CEP 510 P. Albornoz Medina (P. Albornoz Medina) Anastrepha fraterculus [Diptera: Tephritidae] 5 Ene. 10, ARG: Tucumán. Horco Molle, Yerba Buena [SDYA 1/4] [AD, F-20 $0^{\circ} \mathrm{C}$ ]

CEP 526 P. Albornoz Medina (P. Albornoz Medina), Anastrepha fraterculus [Diptera: Tephritidae] 5 Abr. 10, ARG: Tucumán. Horco Molle, Yerba Buena [SDYA 1/4] [AD, F-20 $20^{\circ} \mathrm{C}$

CEP 527 P. Albornoz Medina (P. Albornoz Medina), Anastrepha fraterculus [Diptera: Tephritidae] 5 Abr. 10, ARG: Tucumán. Horco Molle, Yerba Buena [SDYA 1/4] [AD, F-20 $0^{\circ} \mathrm{C}$ ]

CEP 557 J. Tornesello (H.E. Reinoso) Spodoptera frugiperda [Lepidoptera: Noctuidae] 26 Jun. 15 ARG: Buenos Aires, La Plata, CEPAVE [SDYA 1/4] [AD, F-20 ${ }^{\circ} \mathrm{C}$ ]

CEP 558 J. Tornesello (C.C. López Lastra) Spodoptera frugiperda [Lepidoptera: Noctuidae] 7 Jul. 15 ARG: Buenos Aires, La Plata, CEPAVE [SDYA 1/4] [AD, F $-20^{\circ} \mathrm{C}$ ]

\section{Aspergillus fumigatus Fresenius}

CEP 283 G. Marti (G. Marti), Triatoma infestans [Heteroptera: Reduviidae] 17 Dic. 03 ARG: Santiago del Estero, Dto. Giménez, El Quebracho, peridomicilio [AM 2\%] [AD] (*)

\section{Arthrinium sphaerospermum Fuckel}

257 G. Marti (G. Marti \& C.C. López Lastra) Triatoma infestans [Heteroptera: Reduviidae] 1 Ago. 03 ARG: Mendoza [AM 2\%] $[\mathrm{AD}, \mathrm{L}]\left({ }^{*}\right)$

Beauveria bassiana s. I. (Balsamo-Crivelli) Vuilemin

CEP 042 Leg. D. Ohashi (C.C. López Lastra) Crysomelidae NI [Coleoptera: Crysomelidae] 1 May. 02 ARG: Misiones, Cerro Azul [AM, SDYA] [F-20 $\left.0^{\circ} \mathrm{C}, \mathrm{AD}, \mathrm{L}\right]$

CEP 044 [ARSEF 7147; LPS 771] G. Marti (C.C. López Lastra) Triatoma infestans [Heteroptera: Reduvidae] 1 May. 02 ARG: Córdoba [AM, SDYA] [AD, L] (*)

CEP 075 [ARSEF 7466] A. Toledo \& C.C. López Lastra (C.C. López Lastra) Curculionidae NI [Coleoptera Curculionidae] 30 Dic. 03 ARG: Buenos Aires, Berazategui, ECAS [AM, SDYA] $\left[\mathrm{F}-20^{\circ} \mathrm{C}, \mathrm{P}, \mathrm{AD}, \mathrm{L}\right]\left(^{*}\right)$

CEP 077 [ARSEF 7467; LPSC 885] A. Toledo \& C.C. López Lastra (C.C. López Lastra) Balacha melanocephala [Coleoptera: Curculionidae] 24 Feb. 04 ARG: Buenos Aires, Los Hornos, 137 y 89 [AM, SDYA] [F- $\left.20^{\circ} \mathrm{C}, \mathrm{f}-70^{\circ} \mathrm{C}, \mathrm{P}, \mathrm{AD}\right]\left(^{*}\right)$

CEP 081 [ARSEF 7469; LPSC 887] A. Toledo \& C.C. López Lastra (C.C. López Lastra) Balacha melanocephala [Coleoptera: Curculionidae] 20 Mar. 04 ARG: Buenos Aires, San Vicente, zanja Parque San Francisco $[\mathrm{AM}, \mathrm{SDYA}]\left[\mathrm{F}-20^{\circ} \mathrm{C}, \mathrm{AD}\right]\left({ }^{*}\right)$

CEP 091 [LPS 888] A. Toledo \& C.C. López Lastra (A. Toledo \& C.C. López Lastra) Euchistus sp. [Hemiptera: Pentatomidae] 7 Abr. 04 ARG: Buenos Aires, La Plata, Colonia Urquiza [AM, SDYA] [F-20 $\left.20^{\circ}, P, A D, L\right](*)$

CEP 092 [ARSEF 7471] A. Toledo \& C.C. López Lastra (C.C.
López Lastra) Curculionidae NI [Coleoptera: Curculionidae] 7 Abr. 04 ARG: Buenos Aires, La Plata, Colonia Urquiza [AM, SDYA] [P, AD, L] $\left(^{*}\right)$

CEP 112 [ARSEF 8520] A. Toledo \& C.C. López Lastra (A. Toledo) Curculionidae NI [Coleoptera: Curculionidae] 28 Abr. 04 ARG: Buenos Aires, Ruta 36 y 511 [AM, SDYA] [AD] (*) CEP 114 [ARSEF 8522] A. Toledo \& C.C. López Lastra (A. Toledo) Curculionidae NI [Coleoptera: Curculionidae] 28 Abr. 04 ARG: Buenos Aires, Ruta 36 y 511. [AM, SDYA] [P, AD, L] (*) CEP 136 A. Toledo (C.C. López Lastra) Chrysomelidae NI [Coleoptera: Chrysomelidae] 21 Jul. 04 ARG: Buenos Aires, Berazategui, ECAS. [AM, SDYA] [F- $\left.20^{\circ} \mathrm{C}, \mathrm{P}, \mathrm{AD}, \mathrm{L}\right]\left({ }^{*}\right)$ CEP 138 [ARSEF 8461] A. Toledo \& C.C. López Lastra (A. Toledo) Heilipodus erythropus [Coleoptera: Curculionidae] 18 Ago. 04 ARG: Buenos Aires, Magdalena, Bartolomé Bavio, $A^{\circ}$ El Pescado. [AM, SDYA] [F-20 $\left.{ }^{\circ} \mathrm{C}, \mathrm{AD}\right]\left({ }^{*}\right)$

CEP 147 [ARSEF 8372; LPSC 896] A. Toledo (A. Toledo), Cycloneda sanguinea [Coleoptera: Coccinellidae] 11 Mar. 04 ARG: Tucumán, El Manantial [AM, SDYA] $\left[\mathrm{F}-20^{\circ} \mathrm{C}\right]\left({ }^{*}\right)$ CEP 148 [ARSEF 8462] A. Toledo (A. Toledo) Cycloneda sanguinea [Coleoptera: Coccinellidae] 11 Mar. 04 ARG: Tucumán, El Manantial [AM, SDYA] [F-20 $\left.2{ }^{\circ} \mathrm{C}, \mathrm{P}, \mathrm{AD}\right]\left(^{*}\right)$ 149 [ARSEF 8463] A. Toledo (A. Toledo) Cycloneda sanguinea [Coleoptera: Coccinellidae] 11 Mar. 04 ARG: Tucumán, El Manantial [AM, SDYA] [F-20 $\left.{ }^{\circ} \mathrm{C}, \mathrm{P}, \mathrm{AD}, \mathrm{L}\right]$ (*) $^{*}$

CEP 174 S. Cáceres (A.C. Scorsetti) 10 Jun 04 Trialeurodes vaporariorum [Hemiptera: Aleyrodidae] ARG: Corrientes, Bella Vista, INTA [AM $2 \%$, SDYA] [F- $\left.20^{\circ} \mathrm{C}, \mathrm{AD}\right]\left({ }^{*}\right)$

CEP 229 [ARSEF 7776] Leg. A. Briano (C.C. López Lastra) Paysandisia archon [Lepidoptera: Arctridae] 12 Jun. 06 ARG: Bs. As, Baradero [AM, SDYA] [F- $\left.20^{\circ} \mathrm{C}, \mathrm{P}, \mathrm{AD}\right]$

CEP 249 C.C. López Lastra (C.C. López Lastra) de suelo con insecto cebo Tenebrio molitor 18 Dic. 06 ARG: Bs. As., Baradero [AM, SDYA] [F-20 $\left.0^{\circ} \mathrm{C}\right]$

CEP 317 [ARSEF 8929] C. D 'Alessandro (C.C. López Lastra) 25 Jun. 08.

Trialeurodes vaporariorum [Hemiptera: Aleyrodidae] ARG: Buenos Aires. La Plata, Abasto [AM, SDYA] [F-20 $\left.{ }^{\circ} \mathrm{C}, \mathrm{P}, \mathrm{AD}, \mathrm{L}\right]$ CEP 321 P. Albornoz Medina (C.C. López Lastra) 27 Mar. Anastrepha fraterculus [Diptera: Tephritidae] ARG: Tucumán. [AM, SDYA] [P, AD, L]

CEP 322 P. Albornoz Medina (C.C. López Lastra) 27 Mar. 2008 Anastrepha fraterculus [Diptera: Tephritidae] ARG: Tucumán. [AM, SDYA] [P, AD, L]

CEP 323 P. Albornoz Medina, (C.C. López Lastra) 27 Mar. 08 Anastrepha fraterculus [Diptera: Tephritidae] ARG: Tucumán. [AM, SDYA] [P, AD, L]

CEP 324 P. Albornoz Medina (C.C. López Lastra) 27 Mar. 08 Anastrepha fraterculus [Diptera: Tephritidae] ARG: Tucumán [AM, SDYA] [P, AD, L] 
CEP 332 M.E. Schapovaloff (M.E. Schapovaloff \& C.C. López Lastra) aislado de suelo con insecto cebo Tenebrio molitor (suelo cultivado con llex paraguariensis) 1 Nov. 08 ARG: Misiones, Santa Inés [AM, SDYA] $\left[\mathrm{F}-20^{\circ} \mathrm{C}, \mathrm{F}-70^{\circ} \mathrm{C}\right]$

CEP 333 M.E. Schapovaloff (M.E. Schapovaloff \& C.C. López Lastra) aislado de suelo de llex paraguariensis. $1 \mathrm{Nov}$. 08 ARG: Misiones, Santa Inés [AM, SDYA] [F- $\left.20^{\circ} \mathrm{C}, \mathrm{F}-70^{\circ} \mathrm{C}\right]$

CEP 334 M.E. Schapovaloff (M.E. Schapovaloff \& C.C. López Lastra) aislado de suelo cultivado con llex paraguariensis. 1 Nov. 08 ARG: Misiones, Santa Inés [AM, SDYA] [F- $20^{\circ} \mathrm{C}, \mathrm{P}$, $A D, L]$

CEP 335 M.E. Schapovaloff (M.E. Schapovaloff \& C.C. López Lastra) aislado de suelo de llex paraguariensis. 1 Nov. 08 ARG: Misiones, Santa Inés [AM, SDYA] [F-20 $\left.2{ }^{\circ} \mathrm{C}, \mathrm{P}\right]$

CEP 336 M.E. Schapovaloff (M.E. Schapovaloff \& C.C. López Lastra) aislado de suelo con llex paraguariensis. 1 Nov. 08 ARG: Misiones., Santa Inés [AM, SDYA] [F-20 ${ }^{\circ} \mathrm{C}, \mathrm{P}, \mathrm{L}$ ]

CEP 337 M.E. Schapovaloff (M.E. Schapovaloff \& C.C. López Lastra) aislado de suelo de llex paraguariensis. 17 Feb. 09 ARG: Misiones, Santa Inés [AM, SDYA] [F-20 $0^{\circ} \mathrm{C}, \mathrm{AD}$ ]

CEP 338 M.E. Schapovaloff (M.E. Schapovaloff \& C.C. López Lastra) aislado de suelo de llex paraguariensis. 17 Feb. 09 ARG: Misiones, Santa Inés [AM, SDYA] [F- $\left.20^{\circ} \mathrm{C}, \mathrm{F}-70^{\circ} \mathrm{C} P, A D, L\right]$ CEP 339 M.E. Schapovaloff (M.E. Schapovaloff \& C.C. López Lastra) aislado de suelo de llex paraguariensis. 17 Feb. 09 ARG: Misiones, Santa Inés [AM, SDYA] [F-20 $\left.{ }^{\circ} \mathrm{C}, \mathrm{AD}, \mathrm{L}\right]$

CEP 340 M.E. Schapovaloff (M.E. Schapovaloff \& C.C. López Lastra) aislado de suelo de llex paraguariensis. 1 Nov. 08 ARG: Misiones, Santa Inés [AM, SDYA] [F-20 $\left.{ }^{\circ} \mathrm{C}, \mathrm{P}, \mathrm{AD}, \mathrm{L}\right]$

CEP 341 M.E. Schapovaloff (M.E. Schapovaloff \& C.C. López Lastra) aislado de suelo de llex paraguariensis. 17 Feb. 09 ARG: Misiones, Santa Inés [AM, SDYA] [F-20 $\left.{ }^{\circ} \mathrm{C}, \mathrm{P}, \mathrm{AD}, \mathrm{L}\right]$

CEP 342 M.E. Schapovaloff (M.E. Schapovaloff \& C.C. López Lastra) aislado de suelo de llex paraguariensis, 2 Feb. 09 ARG: Misiones, Santa Inés [AM, SDYA] [F-20 $\left.{ }^{\circ} \mathrm{C}, \mathrm{P}, \mathrm{AD}, \mathrm{L}\right]$

CEP 343 M.E. Schapovaloff (M.E. Schapovaloff \& C.C. López Lastra) aislado de suelo de llex paraguariensis. 1 Dic. 08 ARG: Misiones, Santa Inés [AM, SDYA] [F-20 $\left.{ }^{\circ} \mathrm{C}, \mathrm{P}, \mathrm{AD}, \mathrm{L}\right]$

CEP 344 M.E. Schapovaloff (M.E. Schapovaloff \& C.C. López Lastra) aislado de suelo de llex paraguariensis. 1Nov. 08 ARG: Misiones, Santa Inés. [AM, SDYA] [F-20 $\left.{ }^{\circ} \mathrm{C}\right]$

CEP 345 M.E. Schapovaloff (M.E. Schapovaloff \& C.C. López Lastra) aislado de suelo de llex paraguariensis. 1Nov. 08 ARG: Misiones, Santa Inés [AM, SDYA] [F-20 $\left.{ }^{\circ} \mathrm{C}, \mathrm{P}, \mathrm{AD}, \mathrm{L}\right]$

CEP 346 M.E. Schapovaloff (M.E. Schapovaloff \& C.C. López Lastra) aislado de suelo de llex paraguariensis. 17 Feb. 09 ARG: Misiones, Santa Inés [AM, SDYA] [F-20 $\left.{ }^{\circ} \mathrm{C}, \mathrm{P}, \mathrm{AD}, \mathrm{L}\right]$

CEP 347 M.E. Schapovaloff (M.E. Schapovaloff \& C.C. López Lastra) aislado de suelo de llex paraguariensis. 5 May. 09 ARG: Misiones, Santa Inés [AM, SDYA] [F- $\left.20^{\circ} \mathrm{C}, \mathrm{AD}, \mathrm{L}\right]$
CEP 348 M.E. Schapovaloff (M.E. Schapovaloff \& C.C. López Lastra) aislado de suelo de llex paraguariensis. 5 May. 09 ARG: Misiones, Santa Inés [AM, SDYA] [F-20 $\left.{ }^{\circ} \mathrm{C}, \mathrm{AD}, \mathrm{L}\right]$

CEP 417 J. Tornesello (C.C. López Lastra) Tenebrio molitor [Coleoptera: Tenebrionidae] 15 Jun. 13 ARG: Entre Ríos Colón, P. N. El Palmar [AM, SMYA 1\%] [L]

CEP 418 J. Barneche (C.C. López Lastra) Coleoptera N I sobre Bromeliaceae 14 Jun. 13 ARG: Entre Ríos Colón, P. N. El Palmar [AM, SMYA 1\%] [F-70 $\left.{ }^{\circ} \mathrm{C}, \mathrm{L}\right]$

CEP 420 J. Tornesello (C.C. López Lastra) Diabrotica speciosa [Coleoptera: Chrysomelidae] 14 Jun. 13 ARG: Entre Ríos Colón, P. N. El Palmar [AM, SMYA 1\%] [P, L]

CEP 428 Leg. C. Gabellone (C.C. López Lastra) Diabrotica speciosa [Coleoptera: Chrysomelidae] 10 Oct. 14 ARG: Entre Ríos, Colón, P. N. El Palmar [AM, SDYA 1/4] [F-20C, P, AD]

CEP 431 L. Elizalde (C.C. López Lastra) Solenopsis sp. [Hymenoptera: Formicidae] 18 Nov. 14 ARG: Neuquén, Villa La Angostura [YPSS, SMYA $1 \%$ ] [F- $20^{\circ} \mathrm{C}, \mathrm{F}-70^{\circ} \mathrm{C}, \mathrm{AD}$ ]

CEP 432 L. Elizalde (C.C. López Lastra) Solenopsis sp. [Hymenoptera: Formicidae] 18 Nov. 14 ARG: Neuquén, Villa La Angostura [YPSS, SMYA $1 \%$ ] [F- $20^{\circ} \mathrm{C}, \mathrm{F}-70^{\circ} \mathrm{C}, \mathrm{AD}$ ]

CEP 436 J. Barneche (C.C. López Lastra) [Diptera: NI] 1 Jul. 14 ARG: Bs. As. Magdalena, R.P Pearson [YPSS, SMYA 1\%] [F- $\left.20^{\circ} \mathrm{C}, \mathrm{F}-70^{\circ} \mathrm{C}, \mathrm{P}, \mathrm{AD}\right]$

CEP 537 L. Elizalde (C.C. López Lastra) Solenopsis [Hymenoptera: Formicidae] 28 Mar. 14 ARG: Neuquén, Villa Llanquin La Lipela [YPSS, SMYA 1\%] [AD]

CEP 538 L. Elizalde (C.C. López Lastra) Solenopsis [Hymenoptera: Formicidae] 28 Mar. 14 ARG: Neuquén, Villa Llanquín La Lipela [YPSS, SMYA 1\%] [AD]

CEP 539 L. Elizalde (C.C. López Lastra) Solenopsis [Hymenoptera: Formicidae] 28 Mar. 14 ARG: Neuquén, Villa Llanquín, La Lipela [YPSS, SMYA 1\%] [AD]

CEP 540 L. Elizalde (C.C. López Lastra) Solenopsis [Hymenoptera: Formicidae] 28 Mar. 14 ARG: Neuquén, Villa Llanquín La Lipela [YPSS-SMYA 1\%] [AD]

CEP 541 L. Elizalde (C.C. López Lastra) Solenopsis [Hymenoptera: Formicidae] 28 Mar. 14 ARG: [YPSS-SMYA 1\%] [AD]

CEP 545 L. Elizalde (C.C. López Lastra) Acromyrmex lobicornis [Hymenoptera: Formicidae] 28 Mar. 14 ARG: Rio Negro., Pilcaniyeu, Villa Llanquín La Lipela [SMYA $1 / 4]\left[\mathrm{F}-20^{\circ} \mathrm{C}, \mathrm{P}\right.$, $\mathrm{AD}, \mathrm{L}]$

CEP 546 L. Elizalde (C.C. López Lastra) Acromyrmex lobicornis [Hymenoptera: Formicidae] 28 Mar. 14 ARG: Rio Negro, Pilcaniyeu, Villa Llanquín, La Lipela [SDYA $1 / 4]\left[\mathrm{F}-20^{\circ} \mathrm{C}, \mathrm{P}\right.$, $\mathrm{AD}, \mathrm{L}]$

CEP 547 L. Elizalde (C.C. López Lastra) Acromyrmex lobicornis [Hymenoptera: Formicidae] 28 Mar. 14 ARG: Rio Negro, Pilcaniyeu, Villa Llanquin, La Lipela [SDYA 1/4] [F-20 $\left.{ }^{\circ} \mathrm{C}, \mathrm{P}, \mathrm{AD}\right]$ 
CEP 548 L. Elizalde (C.C. López Lastra) Acromyrmex lobicornis [Hymenoptera: Formicidae] 28 Mar. 14 ARG: R. Neg., Pilcaniyeu, Villa Llanquin, La Lipela [SDYA 1/4] [F-20 $\left.{ }^{\circ} \mathrm{C}, \mathrm{P}, \mathrm{AD}\right]$ CEP 549 L. Elizalde (C.C. López Lastra) Acromyrmex lobicornis [Hymenoptera: Formicidae] 28 Mar. 14 ARG: R. Neg., Pilcaniyeu, Villa Llanquín, La Lipela [SDYA 1/4] [P, AD, L] CEP 551 L. Elizalde (C.C. López Lastra) Acromyrmex lobicornis [Hymenoptera: Formicidae] 28 Mar. 14 ARG: Río Negro, Pilcaniyeu, Villa Llanquín, La Lipela [SDYA $1 / 4]\left[\mathrm{F}-20^{\circ} \mathrm{C}\right.$, $\left.\mathrm{F}-70^{\circ} \mathrm{C} P, \mathrm{AD}\right]$

CEP 560 Leg. E. Punschke (C.C. López Lastra) Edesa meditabunda [Hemiptera: Pentatomidae] 22 Oct. 15 ARG: Santa Fe, Zaballa [SDYA 1/4] [P, AD, L]

CEP 561 Leg. E. Punschke (C.C. López Lastra) Edesa meditabunda [Hemiptera: Pentatomidae] 22 Oct. 15 ARG: Santa Fe, Zaballa [SDYA 1/4] [P, AD, L]

CEP 562 Leg. E. Punschke (C.C. López Lastra) Edesa meditabunda [Hemiptera: Pentatomidae] 22 Oct. 15 ARG: Santa Fe, Zaballa [SDYA 1/4] [P, AD]

CEP 563 D. Vera (C.C. López Lastra) aislado de suelo mediante cebo Tenebrio molitor 24 Oct. 15 ARG: Buenos Aires, La Plata [SDYA 1/4] [F-20 $\left.{ }^{\circ} \mathrm{C},-70^{\circ} \mathrm{C}, \mathrm{P}, \mathrm{AD}\right]$

CEP 564 D. Vera (C.C. López Lastra) aislado de suelo mediante cebo Tenebrio molitor 24 Oct. 15 ARG: Buenos Aires, La Plata [SDYA 1/4] [F-20 ${ }^{\circ} \mathrm{C},-70^{\circ} \mathrm{C}, \mathrm{P}, \mathrm{AD}$ ]

CEP 565 D. Vera (C.C. López Lastra) aislado de suelo mediante cebo Tenebrio molitor 24 Oct. 15 ARG: Buenos Aires, La Plata [SDYA 1/4] [F- $\left.20^{\circ} \mathrm{C},-70^{\circ} \mathrm{C}, \mathrm{P}, \mathrm{AD}\right]$

CEP 568 A. Chuchuy (C.C. López Lastra) Acromyrmex lundi [Hymenoptera: Formicidae] 6 Jun. 16 ARG: Buenos Aires, La Plata, Tolosa [SDYA 1/4] [F-70 $\left.{ }^{\circ} \mathrm{C}, \mathrm{P}\right]$

CEP 569 A. Chuchuy (C.C. López Lastra) Acromyrmex lundi [Hymenoptera: Formicidae] 6 Jun. 16 ARG: Buenos Aires, La Plata, Tolosa [SDYA $1 / 4]\left[\mathrm{F}-70^{\circ} \mathrm{C}, \mathrm{P}\right]$

CEP 570 A. Chuchuy (C.C. López Lastra) Acromyrmex lundi [Hymenoptera: Formicidae] 6 Jun. 16 ARG: Buenos Aires, La Plata, Tolosa [SDYA $1 / 4]\left[\mathrm{F}-70^{\circ} \mathrm{C}, \mathrm{P}\right]$

CEP 571 A. Chuchuy (C.C. López Lastra) Acromyrmex lundi [Hymenoptera: Formicidae] 6 Jun. 16 ARG: Buenos Aires, La Plata, Tolosa [SDYA 1/4] [F-70 $\left.{ }^{\circ} \mathrm{C}, \mathrm{P}\right]$

CEP 572 A. Chuchuy (C.C. López Lastra) Acromyrmex lundi [Hymenoptera: Formicidae] 6 Jun. 16 ARG: Buenos Aires, La Plata, Tolosa [SDYA 1/4] [F-70 $\left.{ }^{\circ} \mathrm{C}, \mathrm{P}\right]$

CEP 573 A. Chuchuy (C.C. López Lastra) Acromyrmex lundi [Hymenoptera: Formicidae] 6 Jun. 16 ARG: Buenos Aires, La Plata, Tolosa [SDYA 1/4] [F-70 $\left.{ }^{\circ} \mathrm{C}, \mathrm{P}\right]$

CEP 574 A. Chuchuy (C.C. López Lastra) Acromyrmex lundi [Hymenoptera: Formicidae] 6 Jun. 16 ARG: Buenos Aires, La Plata, Tolosa [SDYA $1 / 4]\left[\mathrm{F}-70^{\circ} \mathrm{C}, \mathrm{P}\right]$

CEP 588 J. Tornesello (C.C. López Lastra) Coleoptera NI, ARG:
Bs. As. La Plata, Bosque 19 Jul. 06 [SDYA 1/4] [F-70 ${ }^{\circ} \mathrm{C}, \mathrm{P}$, $A D, L]$

CEP 601 B. Moreno (C.C. López Lastra) [Hemiptera: Pentatomidae] 22 Nov. 16 ARG: Misiones. PN Iguazú [SDYA 1/4] [P, L]

CEP 603 B. Moreno (C.C. López Lastra) [Hemiptera: Pentatomidae] 24 Nov. 16 ARG: Misiones. PN Iguazú [SDYA 1/4] [P, L]

CEP 606 B. Moreno (C.C. López Lastra) Dinoponera australis [Hemiptera: Pentatomidae] 14 Nov. 16 ARG: Misiones. PP Península [SDYA 1/4] [P, L]

CEP 607 B. Moreno (C.C. López Lastra) [Diptera: Culicidae NI] 8 Nov. 16 ARG: Misiones. PP Urugua-i [SDYA 1/4] [P, L]

CEP 608 B. Moreno (C.C. López Lastra) [Diptera: Muscidae] 10 Nov. 16 ARG: Misiones. PP Urugua-i [SDYA 1/4] [F-70 ${ }^{\circ} \mathrm{C}$, $\mathrm{P}, \mathrm{L}]$

CEP 609 B. Moreno (C.C. López Lastra) Entymus sp. [Coleoptera: Pentatomidae] 15 Nov. 16 ARG: Misiones. PP Península [SDYA 1/4] [P, L]

CEP 612 A. Musso (A. Musso \& G. Dal Bello) 12 Ene. 16 Galleria mellonella, suelo de Fac Agr. ARG: Bs. As. La Plata [SDYA 1/4] [P, L]

CEP 613 A. Musso (A. Musso \& G. Dal Bello) 12 Ene. 16 Galleria mellonella, suelo ARG: Bs. As. La Plata [SDYA 1/4] [P, L]

CEP 614 A. Musso (A. Musso \& G. Dal Bello) 12 Ene. 16 Galleria mellonella, suelo ARG: Bs. As. La Plata [SDYA 1/4] [P, L]

CEP 627 B. Moreno (C.C. López Lastra \& J. Tornesello) 7 Feb. 17 [Lepidoptera] ARG: Jujuy, P.N. Calilegua [SDYA $1 / 4]\left[\mathrm{F}-70^{\circ} \mathrm{C}\right.$, $\mathrm{P}, \mathrm{L}]$

CEP 628 B. Moreno (C.C. López Lastra) 7 Feb. 17 [Lepidoptera] ARG: Jujuy., P.N. Calilegua [SDYA 1/4] [F-70 $\left.{ }^{\circ} \mathrm{C}, \mathrm{P}, \mathrm{L}\right]$

CEP 629 B. Moreno (C.C. López Lastra) [Coleoptera: Cerambycidae] 30 Ene. 17 ARG: Jujuy, P.N. Calilegua [SDYA $1 / 4]\left[\mathrm{F}-70^{\circ} \mathrm{C}, \mathrm{P}, \mathrm{L}\right]$

CEP 630 B. Moreno (C.C. López Lastra) [Hemiptera: Pentatomidae] 30 Ene. 17 ARG: Jujuy, P.N. Calilegua [SDYA 1/4] [F-70 $\mathrm{C}, \mathrm{P}, \mathrm{L}]$

CEP 631 B. Moreno (C.C. López Lastra) [Hemiptera: Cicadidae] 1 Feb. 17 ARG: Jujuy., P.N. Calilegua [SDYA 1/4] [F-70 ${ }^{\circ} \mathrm{C}, \mathrm{P}, \mathrm{L}$ ] CEP 632 B. Moreno (C.C. López Lastra) [Coleoptera: Curculionidae] 13 Feb. 17 ARG: Salta, P.N. El Rey [SDYA 1/4] $\left[\mathrm{F}-70^{\circ} \mathrm{C}, \mathrm{P}, \mathrm{L}\right]$

CEP 633 B. Moreno (C.C. López Lastra) [Hemiptera] 13 Feb. 17 ARG: Salta, P.N. El Rey [SDYA 1/4] [F-70 $\left.{ }^{\circ} \mathrm{C}, \mathrm{P}, \mathrm{L}\right]$

CEP 634 B. Moreno (C.C. López Lastra) [Coleoptera: Curculionidae] 13 Feb. 17 ARG: Salta, P.N. El Rey [SDYA 1/4] $\left[\mathrm{F}-70^{\circ} \mathrm{C}, \mathrm{P}, \mathrm{L}\right]$

CEP 635 B. Moreno (C.C. López Lastra) [Lepidoptera] 14 Feb. 
17 ARG: Salta, P.N. El Rey [SDYA $1 / 4]$ ] $\left[F-70^{\circ} \mathrm{C}, \mathrm{P}, \mathrm{L}\right]$

CEP 636 B. Moreno (C.C. López Lastra) [Hemiptera: Pentatomidae] 17 Feb. 17 ARG: Salta, P.N. El Rey [SDYA 1/4] $\left[\mathrm{F}-70^{\circ} \mathrm{C}, \mathrm{P}, \mathrm{L}\right]$

CEP 637 B. Moreno (C.C. López Lastra) [Lepidoptera] 17 Feb. 17 ARG: Salta, P.N. El Rey [SDYA 1/4] [F-70 C, P, L]

CEP 638 B. Moreno (C.C. López Lastra) [Hymenoptera] 17 Feb. 17 ARG: Salta, P.N. El Rey [SDYA $1 / 4$ ] [F-70 ${ }^{\circ} \mathrm{C}, \mathrm{P}, \mathrm{L}$ ]

CEP 639 B. Moreno (C.C. López Lastra) [Lepidoptera] 17 Feb. 17 ARG: Salta, P.N. El Rey [SDYA $1 / 1 / 4]\left[F-70^{\circ} \mathrm{C}, \mathrm{P}, \mathrm{L}\right]$

CEP 640 B. Moreno (C.C. López Lastra) [Coleoptera: Cerambycidae] 17 Feb. 17 ARG: Salta, P.N. El Rey [SDYA 1/4] $\left[\mathrm{F}-70^{\circ} \mathrm{C}, \mathrm{P}, \mathrm{L}\right]$

CEP 641 B. Moreno (C.C. López Lastra) [Coleoptera: Curculionidae] 17 Feb. 17 ARG: Salta, P.N. El Rey [SDYA 1/4] $\left[\mathrm{F}-70^{\circ} \mathrm{C}, \mathrm{P}, \mathrm{L}\right]$

CEP 642 B. Moreno (C.C. López Lastra) [Lepidoptera] 18 Feb. 17 ARG: Salta, P.N. El Rey [SDYA 1/4] [F-70 C, P, L]

CEP 644 B. Moreno (C.C. López Lastra) [Hemiptera: Pentatomidae] 18 Feb. 17 ARG: Jujuy, P.N. Calilegua [SDYA $1 / 4]\left[\mathrm{F}-70^{\circ} \mathrm{C}, \mathrm{P}, \mathrm{L}\right]$

CEP 650 B. Moreno (C.C. López Lastra) [Hymenoptera: Formicidae] 9 Abr. 17 ARG: Misiones., P.P. Península [SDYA $1 / 4]\left[\mathrm{F}-70^{\circ} \mathrm{C}, \mathrm{P}, \mathrm{L}\right]$

CEP 653 B. Moreno (C.C. López Lastra) [Hymenoptera: Formicidae] 9 Abr. 17 ARG: Misiones., P.P. Península [SDYA $1 / 4]\left[\mathrm{F}-70^{\circ} \mathrm{C}, \mathrm{P}, \mathrm{L}\right]$

CEP 662 B. Moreno (C.C. López Lastra) [Hymenoptera: Formicidae] 9 Abr. 17 ARG: Misiones., P.P. Península [SDYA $1 / 4]\left[\mathrm{F}-70^{\circ} \mathrm{C}, \mathrm{P}, \mathrm{L}\right]$

CEP 674 B. Moreno (C.C. López Lastra) [Hymenoptera: Formicidae] 9 Abr. 17 ARG: Misiones., P.P. Península [SDYA $1 / 4]\left[\mathrm{F}-70^{\circ} \mathrm{C}, \mathrm{P}, \mathrm{L}\right]$

CEP 675 B. Moreno (C.C. López Lastra) [Hymenoptera: Formicidae] 9 Abr. 17 ARG: Misiones., P.P. Península [SDYA $1 / 4]\left[\mathrm{F}-70^{\circ} \mathrm{C}, \mathrm{P}, \mathrm{L}\right]$

CEP 685 A. Musso (A. Musso) Galleria melonella [Lepidoptera: Pyralidae] 18 Jul. 17 ARG: Bs As. Est. Exp. Los Hornos, FCAyF [SDYA $1 / 4]\left[\mathrm{F}-70^{\circ} \mathrm{C}, \mathrm{P}, \mathrm{L}\right]$

CEP 686 A. Musso (A. Musso) Galleria melonella [Lepidoptera: Pyralidae] 18 Jul. 17 ARG: Bs As. Est. Exp. Los Hornos, FCAyF [SDYA $1 / 4]\left[\mathrm{F}-70^{\circ} \mathrm{C}, \mathrm{P}, \mathrm{L}\right]$

Clonostachys rosea (Link: Fries) Schroers, Samuels, Seifert \& W. Gams

CEP 066 A. Toledo (C.C. López Lastra) [ARSEF 7472, CBS 115883] Sonesimia grossa [Hemiptera: Cicadellidae] 27 Sep. O3E Ríos, Colón, Parque Nacional El Palmar.*

Conidiobolus coronatus (Costatin) A. Batko *

CEP 062 [ARSEF 7203] A. Toledo \& C.C. López Lastra (C.C.
López Lastra) Delphacodes haywardi [Hemiptera: Delphacidae] 15 Ago. 03 ARG: Buenos Aires, La Plata [SDYA] [F-70 $\left.{ }^{\circ} \mathrm{C}\right]^{*}$

Fusarium sp.

CEP 483 Albornoz Medina (M. Cabello) Aphid [Hemiptera: Aphidoidea] 15 May. 06 ARG: Tucumán, Horco Molle [SDYA 1/4] [F-20 $\left.{ }^{\circ} \mathrm{C}\right]$

CEP 485 P. Albornoz Medina, (M. Cabello) Anastrepha sp. [Diptera: Tephritidae] 29 Ago. 14 ARG: Tucumán, Horco Molle [SDYA 1/4] [F-20 $\left.{ }^{\circ} \mathrm{C}\right]$

CEP 486 P. Albornoz Medina, (M. Cabello), Anastrepha sp. [Diptera: Tephritidae] 29 Ago. 14 ARG: Tucumán, Horco Molle [SDYA $1 / 4]\left[\mathrm{F}-20^{\circ} \mathrm{C}\right]$

CEP 496 P. Albornoz Medina, Anastrepha sp. [Diptera: Tephritidae] 17 Ago. 14 ARG: Tucumán, Horco Molle [SDYA 1/4] [F-20 $\left.{ }^{\circ} \mathrm{C}\right]$

CEP 499 P. Albornoz Medina (M. Cabello) Anastrepha fraterculus [Diptera: Tephritidae] 29 Ago. 14 ARG: Tucumán, Horco Molle [SDYA 1/4] [F-20 $\left.{ }^{\circ} \mathrm{C}\right]$

CEP 500 P. Albornoz Medina (M. Cabello) Anastrepha fraterculus [Diptera: Tephritidae] 29 Ago. 14 ARG: Tucumán, Horco Molle [SDYA $1 / 4]\left[\mathrm{F}-20^{\circ} \mathrm{C}\right]$

CEP $503\left[\mathrm{~F}-20^{\circ} \mathrm{C}\right]$ P. Albornoz Medina (M. Cabello) Anastrepha fraterculus [Diptera: Tephritidae] 29 Ago. 14 ARG: Tucumán, Horco Molle [SDYA 1/4] [F-20 $\mathrm{C}$ ]

CEP 552 P. Albornoz Medina (M. Cabello) Anastrepha fraterculus [Diptera: Tephritidae] 29 Ago. 14 ARG: Tucumán, Horco Molle [SDYA $1 / 4$ [F- $\left.20^{\circ} \mathrm{C}\right]$

CEP 554 J. Barneche (C.C. López Lastra) Stenoterommata platensis [Araneae: Nemesiidae] 20 Jun. 14 ARG: Bs. As., Magdalena, Reserva Pearson [SDYA 1/4] [AD]

\section{Isaria sp.}

CEP 049 M. Murace (C.C. López Lastra) [Hymenoptera: Vespidae] 1 Nov. 98 ARG: Buenos Aires, (Delta) San Fernando [AM, SDYA] [F-20 $\left.{ }^{\circ} \mathrm{C}\right] *$

CEP 566 D. Vera (C.C. López Lastra) Tenebrio molitor (cebo) tierra domiciliaria enriquecida con abono 24 Oct. 15 ARG: Buenos Aires, La Plata [SDYA 1/4] [F-70 $\mathrm{C}, \mathrm{P}, \mathrm{L}]$

Isaria farinosa (Holmsk.) Fr.

CEP 027 C.C. López Lastra (C.C. López Lastra \& A.C. Scorsetti) Trialeurodes vaporariorum [Hemiptera: Aleyloridae] 1 Jun. 01 ARG: Buenos Aires, La Plata, Cnia. Urquiza. [AM, SDYA] $\left[\mathrm{F}-20^{\circ} \mathrm{C}\right]\left({ }^{*}\right)$

\section{Isaria fumosorosea Wize}

CEP 029 [ARSEF 6717; LPS 770] C.C. López Lastra (C.C. López Lastra \& A. C. Scorsetti) Trialeurodes vaporariorum [Hemiptera: Aleyrodidae] 1 May. 01 ARG: Buenos Aires, La Plata, Cnia Urquiza. [AM, SDYA] $\left[\mathrm{F}-20^{\circ} \mathrm{C}\right]\left({ }^{*}\right)(* *)$ 
CEP 031 [ARSEF 7050; LPS 769] C.C. López Lastra (C.C. López Lastra \& A.Toledo) Oliarus dimidiatus [Hemiptera: Cixiidae] 1 Nov. 01 ARG: Buenos Aires, La Plata, [AM, SDYA] [AD] (*) CEP 032 [ARSEF 7051] C.C. López Lastra (C.C. López Lastra \& A. C. Scorsetti) Trialeurodes vaporariorum [Hemiptera: Aleyrodidae] 1 Mar. 02 ARG: Buenos Aires, La Plata, Cnia Urquiza. [AM, SDYA] [AD, L] (*)

CEP 302 [ARSEF 8716] C. D’Alessandro (C.C. López Lastra) Trialeurodes vaporariorum [Hemiptera: Aleyroridae] 22 May. 07 ARG: Buenos Aires, La Plata, [AM, SDYA] [F-20 $2{ }^{\circ} \mathrm{C}\left({ }^{*}\right)\left({ }^{* *}\right)$ CEP 303 [ARSEF 8717] C. D’Alessandro (C.C. López Lastra) Trialeurodes vaporariorum [Hemiptera: Aleyrodidae] 4 Jun. 07 ARG: Buenos Aires, La Plata, Abasto [AM, SDYA] [F- $20^{\circ} \mathrm{C}, \mathrm{P}$, $\mathrm{AD}]\left({ }^{*}\right)\left({ }^{* *}\right)$

CEP 304 [ARSEF 8718] C. D’Alessandro (C.C. López Lastra) Trialeurodes vaporariorum [Hemiptera: Aleyrodidae] 3 Oct. 07 ARG: Buenos Aires, La Plata, Cnia. Urquiza [AM, SDYA] $\left[\mathrm{F}-20^{\circ} \mathrm{C}, \mathrm{AD}\right]\left({ }^{*}\right)\left({ }^{* *}\right)$

CEP 305 [ARSEF 8719] C. D’Alessandro (C.C. López Lastra) Trialeurodes vaporariorum [Hemiptera: Aleyrodidae] 3 Oct. 07 ARG: Buenos Aires, La Plata, Cnia. Urquiza [AM, SDYA] $\left[\mathrm{F}-20^{\circ} \mathrm{C}, \mathrm{P}, \mathrm{L}\right]\left(^{*}\right)\left(^{* *}\right)$

CEP 306 [ARSEF 8720] C. D’Alessandro (C.C. López Lastra) Trialeurodes vaporariorum [Hemiptera: Aleyrodidae] 10 Oct. 07 ARG: Buenos Aires, La Plata, Cnia. Urquiza [AM, SDYA] $\left[\mathrm{F}-20^{\circ} \mathrm{C}, \mathrm{P}, \mathrm{AD}, \mathrm{L}\right]\left(^{*}\right)\left(^{* *}\right)$

CEP 307 [ARSEF 8721] C. D’Alessandro (C.C. López Lastra) Trialeurodes vaporariorum [Hemiptera: Aleyrodidae] 16 Oct. 07 ARG: Buenos Aires, La Plata, Cnia. Urquiza [AM, SDYA] $\left[\mathrm{F}-20^{\circ} \mathrm{C}, \mathrm{P}, \mathrm{AD}, \mathrm{L}\right]\left({ }^{*}\right)\left(^{* *}\right)$

CEP 308 [ARSEF 8723] C. D’Alessandro (C.C. López Lastra) Trialeurodes vaporariorum [Hemiptera: Aleyrodidae] 4 Jun. 07 ARG: Buenos Aires, La Plata, Cnia. Urquiza [AM, SDYA] $\left[\mathrm{F}-20^{\circ} \mathrm{C}, \mathrm{P}, \mathrm{AD}\right]\left(^{*}\right)\left(^{* *}\right)$

CEP 309 [ARSEF 8724] C. D’Alessandro (C.C. López Lastra) Trialeurodes vaporariorum [Hemiptera: Aleyrodidae] 6 Jun. 07 ARG: Buenos Aires, La Plata, Cnia. Urquiza [AM, SDYA] $\left[\mathrm{F}-20^{\circ} \mathrm{C}, \mathrm{P}, \mathrm{AD}, \mathrm{L}\right]\left(^{*}\right)\left({ }^{* *}\right)$

CEP 310 [ARSEF 8725] C. D’Alessandro (C.C. López Lastra) Trialeurodes vaporariorum [Hemiptera: Aleyrodidae] 6 Jun. 07 ARG: Buenos Aires, La Plata, Cnia. Urquiza [AM, SDYA] $\left[\mathrm{F}-20^{\circ} \mathrm{C}, \mathrm{P}, \mathrm{AD}\right]\left({ }^{*}\right)\left(^{* *}\right)$

CEP 311 [ARSEF 8726] C. D’Alessandro (C.C. López Lastra) Trialeurodes vaporariorum [Hemiptera: Aleyrodidae] 6 Jun. 07 ARG: Buenos Aires, La Plata, Cnia. Urquiza [AM, SDYA] $\left[\mathrm{F}-20^{\circ} \mathrm{C}\right]\left({ }^{*}\right)\left({ }^{* *}\right)$

CEP 312 [ARSEF 8727] C. D’Alessandro (C.C. López Lastra) Trialeurodes vaporariorum [Hemiptera: Aleyrodidae] 13 Jun. 07 ARG: Buenos Aires, La Plata, Cnia. Urquiza [AM, SDYA] $\left[\mathrm{F}-20^{\circ} \mathrm{C}\right]\left({ }^{*}\right)\left({ }^{* *}\right)$
CEP 313 [ARSEF 8728] C. D’Alessandro (C.C. López Lastra) Trialeurodes vaporariorum [Hemiptera: Aleyrodidae] 13 Jun. 07 ARG: Buenos Aires, La Plata, Cnia. Urquiza [AM, SDYA] $\left[\mathrm{F}-20^{\circ} \mathrm{C}, \mathrm{P}, \mathrm{AD}\right]\left({ }^{*}\right)(* *)$

CEP 314 [ARSEF 8729] C. D’Alessandro (C.C. López Lastra) Trialeurodes vaporariorum [Hemiptera: Aleyrodidae] 22 May. 07 ARG: Buenos Aires, La Plata, Cnia. Urquiza [AM, SDYA] $\left[\mathrm{F}-20^{\circ} \mathrm{C}, \mathrm{P}\right]\left({ }^{*}\right)\left(^{* *}\right)$

CEP 315 [ARSEF 8730] C. D’Alessandro (C.C. López Lastra) Trialeurodes vaporariorum [Hemiptera: Aleyroridae] 22 May. 07 ARG: Buenos Aires, La Plata, Cnia. Urquiza [AM, SDYA] $\left[\mathrm{F}-20^{\circ} \mathrm{C}, \mathrm{P}, \mathrm{AD}, \mathrm{L}\right]\left(^{*}\right)$

CEP 316 [ARSEF 8928] C. D’Alessandro (C.C. López Lastra) Trialeurodes vaporariorum [Hemiptera: Aleyrodidae] 22 May. 07 ARG: Buenos Aires, La Plata, Cnia. Urquiza [AM, SDYA] $\left[\mathrm{F}-20^{\circ} \mathrm{C}, \mathrm{P}, \mathrm{AD}, \mathrm{L}\right]\left(^{*}\right)$

Isaria tenuipes Peck

CEP 425 J. Aguilera Sammaritano (C.C. López Lastra). [Phasmidae ] NI "bicho palo", 22 Ago. 14 ARG: Entre Ríos, Colón, P.N. El Palmar [SDYA 1/4] [F-20, P, AD, L]

\section{Lecanicillium $\mathrm{sp}$.}

CEP 419 C.C. López Lastra (C.C. López Lastra) Coccidae NI [Hymenoptera: Coccidae] 15 Jun. 13 ARG: Entre Ríos, Colón, Parque Nacional El Palmar [AM, SDYA] [F-20 $\left.20^{\circ} \mathrm{C}, \mathrm{P}, \mathrm{AD}, \mathrm{L}\right]\left(^{*}\right)\left({ }^{* *}\right)$

Lecanicillium aphanocladii Zare \& W. Gams

CEP 553 J. Barneche (C.C. López Lastra), Stenoterommata platensis [Araneae: Nemesiidae] 20 Jun. 14 ARG: Buenos Aires, Magdalena, Reserva Pearson [SDYA 1/4] [F-20 $\left.{ }^{\circ} \mathrm{C}\right]\left({ }^{*}\right)$ CEP 556 J. Barneche (C.C. López Lastra) Stenoterommata platensis [Araneae: Nemesidae] 20 Jun. 14 ARG: Buenos Aires, La Plata, CEPAVE [AM, SDYA] [F- $\left.70^{\circ} \mathrm{C}, \mathrm{AD}\right]$

Lecanicillium lecanii (Zimm.) Zare \& W. Gams

CEP 057 [ARSEF 7207] A.C. Scorsetti \& C.C. López Lastra (C.C. López Lastra) Trialeurodes vaporariorum [Hemiptera: Aleyrodidae] 7 Jul. 07 ARG: Buenos Aires, La Plata, El Peligro [AM, SDYA] [F-20 $\left.20^{\circ}, \mathrm{P}, \mathrm{AD}, \mathrm{L}\right]\left({ }^{*}\right)\left(^{* *}\right)$

CEP 108 [ARSEF 7462] A.C. Scorsetti (C.C. López Lastra) Myzus persicae [Hemiptera: Aphididae] 25 Abr. 04 ARG: Buenos Aires, La Plata [AM, SDYA] [F- $\left.20^{\circ} \mathrm{C}, \mathrm{AD}\right]\left({ }^{*}\right)(* *)$ CEP 109 [A.C. Scorsetti (A.C. Scorsetti) Myzus persicae [Hemiptera: Aphididae] 25 Abr. 04 ARG: Buenos Aires, La Plata [AM, SDYA] [AD] $\left({ }^{*}\right)\left({ }^{* *}\right)$

CEP 368 L. Correa (C.C. López Lastra) Ceroplastes grandis [Hemiptera: Aphididae] 15 Ago. 12 ARG: Buenos Aires La Plata, [AM, SDYA] [P, AD]

Lecanicillium longisporum (Petch) Zare \& W. Gams CEP 056 [ARSEF 7206] A.C. Scorsetti \& C.C. López Lastra 
(A.C. Scorsetti \& C.C. López Lastra) Trialeurodes vaporariorum [Hemiptera: Aleyrodidae] 9 Jun 03 ARG: Buenos Aires, La Plata, El Peligro [AM, SDYA] $\left[\mathrm{F}-20^{\circ} \mathrm{C}, \mathrm{F}-20^{\circ} \mathrm{C}, \mathrm{P}, \mathrm{L}\right]\left({ }^{*}\right)\left({ }^{* *}\right)$

CEP 104 [A.C. Scorsetti \& C.C. López Lastra (A.C. Scorsetti \& C.C. López Lastra) Capitophorus elaeagni [Hemiptera: Aphididae] 16 Mar. 04 ARG: Buenos Aires, Bavio, Escuela experimental [AM, SDYA] [AD] $\left({ }^{*}\right)(* *)$

CEP 155 [ARSEF 7459] A.C. Scorsetti (A.C. Scorsetti) Capitophorus elaeagni [Hemiptera: Aphididae] 18 Ago. 04 ARG: Buenos Aires, Magdalena, B. Bavio, Escuela experimental [AM, SDYA] [F-20 $\mathrm{C}, \mathrm{P}, \mathrm{AD}, \mathrm{L}]\left({ }^{*}\right)\left(^{* *}\right)$

CEP 190 [ARSEF 7784] A.C. Scorsetti (C.C. López Lastra) Capitophorus elaeagni [Hemiptera: Aphididae] 22 Mar. 05 ARG: Buenos Aires, Magdalena, General Mansilla, [AM, SDYA] $\left[\mathrm{F}-20^{\circ} \mathrm{C}, \mathrm{P}, \mathrm{AD}\right]\left({ }^{*}\right)(* *)$

\section{Lecanicillium muscarium (Petch) Zare \& W. Gams}

CEP 054 [ARSEF 7460] A.C. Scorsetti \& C.C. López Lastra (A.C. Scorsetti \& C.C. López Lastra) Trialuerodes vaporiorum [Hemiptera: Aleyrodidae] 9 Jun. 03 ARG: Buenos Aires, La Plata, El Peligro [AM, SDYA] [AD] $\left({ }^{*}\right)(* *)$

CEP 063 [ARSEF 7781; LPS 903] A. Toledo (C.C. López Lastra) Delphacodes kuschelli [Hemiptera: Delphacidae] 28 Ago. 03 ARG: Buenos Aires, La Plata, Invernáculo CEPAVE [SDYA] $\left[\mathrm{F}-20^{\circ} \mathrm{C}, \mathrm{P}, \mathrm{AD}, \mathrm{L}\right]\left({ }^{*}\right)\left(^{* *}\right)$

CEP 182 [ARSEF 7782] A.C. Scorsetti (C.C. López Lastra) Myzus persicae [Hemiptera: Aphididae] 29 Dic. 04 ARG: Buenos Aires, La Plata [SDYA] [F-20 $\left.{ }^{\circ} \mathrm{C}, \mathrm{P}, \mathrm{AD}\right]\left({ }^{*}\right)\left({ }^{* *}\right)$ CEP 194 [ARSEF 7785] A.C. Scorsetti (C.C. López Lastra) Aphis fabae [Hymenoptera: Apidae] 22 Mar. 05 ARG: Buenos Aires, Magdalena, General Mansilla [SDYA] [F-20 $\left.20^{\circ}, \mathrm{PAD}\right]\left({ }^{*}\right)\left({ }^{* *}\right)$

\section{Leptolegnia chapmanii (Seymour)}

CEP 010 [ARSEF 5499] J.J. García. (C.C. López Lastra) Ochleroratus albifasciatus [Diptera: Culicidae] 25 Sep. 96 ARG: Buenos Aires, La Plata, Melchor Romero [YPSS; SMYA 1\%] $\left[\mathrm{F}-70^{\circ} \mathrm{C}, \mathrm{AD}, \mathrm{L}\right]\left(^{*}\right)\left(^{* *}\right)$

CEP 433 V. Micieli (C.C. López Lastra) Ochleroratus albifasciatus [Diptera: Culicidae] 10 Ago. 14 ARG: Misiones [SDYA] [F- $\left.20^{\circ} \mathrm{C}, \mathrm{F}-70^{\circ} \mathrm{C}\right]$

Metharizium argentinense Gutierrez, Leclerque \& López Lastra CEP 414 J. Barneche (A. Gutierrez) Blattodea NI 30 Ago. 13 ARG: E. Ríos, El Palmar [APG, SDYA 1\%] [F-20 $\left.2{ }^{\circ} \mathrm{C}, \mathrm{P}\right]\left({ }^{*}\right)\left({ }^{* *}\right)$ CEP 424 J. Barneche (A. Gutierrez) Blattodea [Blaberidae: Epilamprinae] 15 Jun. 14 ARG: Buenos Aires, Magdalena, Reserva Pearson [APG, SDYA 1\%] [P, AD] $\left({ }^{*}\right)\left({ }^{* *}\right)$

Metharizium anisopliae (Metchnikoff) Sorokin

CEP 085 A. Toledo \& C.C. López Lastra (A. Toledo \& C.C. López Lastra) Eryngium sp. 30 Mar. 04 ARG: Buenos Aires, Los
Hornos [AM, SDYA 1] $\left[\mathrm{F}-20^{\circ} \mathrm{C}, \mathrm{F}-70^{\circ} \mathrm{C}, \mathrm{AD}, \mathrm{L}\right]\left({ }^{*}\right)\left({ }^{* *}\right)$ CEP 087 A. Toledo \& C.C. López Lastra (A. Toledo \& C.C. López Lastra) Eryngium sp. 7 Abr. 04 ARG: Buenos Aires, La Plata, Cnia Urquiza Ruta 36 y $511\left[\mathrm{~F}-20^{\circ} \mathrm{C}, \mathrm{F}-70^{\circ} \mathrm{C}, \mathrm{P}, \mathrm{AD}, \mathrm{L}\right]$ $(*)(* *)$

CEP 120 A. Toledo \& C.C. López Lastra (A. Toledo \& C.C. López Lastra) Kanaima fluvialis [Hemiptera: Cercopidae] 12 Abr. 04 ARG: Buenos Aires, La Plata, Cnia Urquiza Ruta 36 y 511 [AM, SDYA 1\%] [F-20 $\left.2{ }^{\circ} \mathrm{C}, \mathrm{F}-70^{\circ} \mathrm{C}, \mathrm{P}, \mathrm{AD}, \mathrm{L}\right]\left(^{(*)}\left(^{* *}\right)\right.$

CEP 122 [ARSEF 8527] A. Toledo \& C.C. López Lastra (A. Toledo) Cercopidae N I [Hemiptera: Cercopidae] 18 Abr. 04 ARG: Buenos Aires, La Plata, Cnia Urquiza Ruta 36 y 511 [AM, SDYA $1 \%]\left[\mathrm{F}-20^{\circ} \mathrm{C}\right]\left({ }^{*}\right)(* *)$

CEP 160 [ARSEF 8376; LPS 908] A. Marino, A. Toledo \& C.C. López Lastra (A. Toledo) Cercopidae N I [Hemiptera: Cercopidae] 6 Oct. 04 ARG: Buenos Aires, Corrientes, Esteros del Iberá [AM, SDYA 1\%] [F-70 $\left.{ }^{\circ} \mathrm{C}, \mathrm{P}, \mathrm{AD}, \mathrm{L}\right]\left(^{*}\right)\left(^{* *}\right)$

CEP 350 M.E. Schapovaloff (M.E. Schapovaloff \& C.C. López Lastra) suelo, 14 May. 2010. ARG Misiones, Campo Ramón sitio 15 [YPSS; SMYA 1\%] [F-20 $\left.2{ }^{\circ} \mathrm{C}, \mathrm{F}-70^{\circ} \mathrm{C}, \mathrm{P}, \mathrm{AD}, \mathrm{L}\right]\left(^{*}\right)\left({ }^{* *}\right)$

CEP 372 J. Aguilera (C.C. López Lastra) Tenebrio molitor [Coleoptera: Tenebrionidae] 15 Sep. 12 ARG: San Juan, Finca Miguel Mas [YPSS, SMYA $1 \%]\left[\mathrm{F}-20^{\circ} \mathrm{C}\right]\left({ }^{*}\right)$

CEP 373 J. Aguilera (C.C. López Lastra) Tenebrio molitor [Coleoptera: Tenebrionidae] 15 Sep. 12 ARG: San Juan, Finca Miguel Mas [YPSS, SMYA $1 \%]\left[\mathrm{F}-20^{\circ} \mathrm{C}\right]\left({ }^{*}\right)$

CEP 374 J. Aguilera (C.C. López Lastra) Tenebrio molitor [Coleoptera: Tenebrionidae] 15 Sep. 12 ARG: San Juan, Pocito Experimental INTA [YPSS, SMYA $1 \%]\left[\mathrm{F}-20^{\circ} \mathrm{C}\right]\left({ }^{*}\right)$

CEP 375 J. Aguilera (C.C. López Lastra) Tenebrio molitor [Coleoptera: Tenebrionidae] 15 Sep. 12 ARG: San Juan, Finca Miguel Mas [YPSS, SMYA $1 \%]\left[\mathrm{F}-20^{\circ} \mathrm{C}\right]\left({ }^{*}\right)$

CEP 376 J. Aguilera (C.C. López Lastra) Tenebrio molitor [Coleoptera: Tenebrionidae] 15 Sep. 12 ARG San Juan, Finca Miguel Más [YPSS, SMYA $1 \%]\left[\mathrm{F}-20^{\circ} \mathrm{C}\right]\left(^{*}\right)$

CEP 377 J. Aguilera (C.C. López Lastra) Tenebrio molitor [Coleoptera: Tenebrionidae] 15 Sep. 12 ARG: San Juan, Finca Miguel Mas [YPSS; SMYA $1 \%$ ] [F-20 $\left.0^{\circ} \mathrm{C}\right]$

CEP 378 J. Aguilera (C.C. López Lastra) Tenebrio molitor [Coleoptera: Tenebrionidae] 20 Sep. 12 ARG: San Juan, Finca Miguel Mas [YPSS, SMYA $1 \%]\left[\mathrm{F}-20^{\circ} \mathrm{C}\right]\left(^{*}\right)$

CEP 379 J. Aguilera (C.C. López Lastra) Tenebrio molitor [Coleoptera: Tenebrionidae] 20 Sep. 12 ARG: San Juan, Finca Miguel Mas [YPSS, SMYA $1 \%]\left[\mathrm{F}-20^{\circ} \mathrm{C}\right]\left(^{*}\right)$

CEP 380 J. Aguilera (C.C. López Lastra) Tenebrio molitor [Coleoptera: Tenebrionidae] 20 Sep. 12 ARG: San Juan, Pocito Experimental INTA [YPSS, SMYA $1 \%]\left[\mathrm{F}-20^{\circ} \mathrm{C}\right]\left({ }^{*}\right)$

CEP 381 J. Aguilera (C.C. López Lastra) Tenebrio molitor [Coleoptera: Tenebrionidae] 20 Sep. 12 ARG: San Juan, Finca Tía Nora [YPSS, SMYA 1\%] [F- $20^{\circ} \mathrm{C}$ ] 
CEP 382 J. Aguilera (C.C. López Lastra) Tenebrio molitor [Coleoptera: Tenebrionidae] 20 Sep. 12 ARG: San Juan, Finca Miguel Mas [YPSS, SMYA $1 \%]\left[\mathrm{F}-20^{\circ} \mathrm{C}\right]\left(^{*}\right)$

CEP 383 J. Aguilera (C.C. López Lastra) Tenebrio molitor [Coleoptera: Tenebrionidae] 1 Oct. 12 ARG: San Juan, Pocito Experimental INTA [YPSS, SMYA $1 \%]\left[\mathrm{F}-20^{\circ} \mathrm{C}\right]\left(^{*}\right)$

CEP 384 J. Aguilera (C.C. López Lastra) Tenebrio molitor [Coleoptera: Tenebrionidae] 1 Oct. 12 ARG: San Juan, Pocito Experimental INTA [YPSS, SMYA $1 \%]\left[\mathrm{F}-20^{\circ} \mathrm{C}\right]\left({ }^{*}\right)$

CEP 385 J. Aguilera (C.C. López Lastra) Tenebrio molitor [Coleoptera: Tenebrionidae] 1 Oct. 12 ARG: San Juan, Finca Miguel Mas [YPSS, SMYA $1 \%]\left[\mathrm{F}-20^{\circ} \mathrm{C}\right]\left(^{*}\right)$

CEP 386 J. Aguilera (C.C. López Lastra) Tenebrio molitor [Coleoptera: Tenebrionidae] 1 Oct. 12 ARG: San Juan, Finca Miguel Mas [YPSS, SMYA $1 \%]\left[\mathrm{F}-20^{\circ} \mathrm{C}\right]\left(^{*}\right)$

CEP 387 J. Aguilera (C.C. López Lastra) Tenebrio molitor [Coleoptera: Tenebrionidae] 1 Oct. 12 ARG: San Juan, Finca Miguel Mas [YPSS, SMYA $1 \%]\left[\mathrm{F}-20^{\circ} \mathrm{C}\right]\left(^{*}\right)$

CEP 388 J. Aguilera (C.C. López Lastra) Tenebrio molitor [Coleoptera: Tenebrionidae] 1 Oct. 12 ARG: San Juan Pocito Experimental INTA [YPSS, SMYA $1 \%]\left[\mathrm{F}-20^{\circ} \mathrm{C}\right]\left({ }^{*}\right)$

CEP 389 J. Aguilera (C.C. López Lastra) Tenebrio molitor [Coleoptera: Tenebrionidae] 1 Oct. 12 ARG: San Juan, Finca Miguel Mas [YPSS, SMYA $1 \%]\left[\mathrm{F}-20^{\circ} \mathrm{C}, \mathrm{F}-70^{\circ} \mathrm{C}\right]\left({ }^{*}\right)$

CEP 390 J. Aguilera (C.C. López Lastra) Tenebrio molitor [Coleoptera: Tenebrionidae] 16 Oct. 12 ARG: San Juan, Finca Miguel Mas [YPSS, SMYA $1 \%]\left[\mathrm{F}-20^{\circ} \mathrm{C}, \mathrm{F}-70^{\circ} \mathrm{C}\right]\left({ }^{*}\right)$

CEP 392 J. Aguilera (C.C. López Lastra) Tenebrio molitor [Coleoptera: Tenebrionidae] 16 Oct. 12 ARG: San Juan Pocito Experimental INTA [YPSS, SMYA $1 \%]\left[\mathrm{F}-20^{\circ} \mathrm{C}\right]\left({ }^{*}\right)$

CEP 393 J. Aguilera (C.C. López Lastra) Tenebrio molitor [Coleoptera: Tenebrionidae] 16 Oct. 12 ARG: San Juan, Finca Miguel Mas [YPSS, SMYA $1 \%$ ] [F- $\left.20^{\circ} \mathrm{C}, \mathrm{F}-70^{\circ} \mathrm{C}, \mathrm{L}\right]$

CEP 395 J. Aguilera (C.C. López Lastra) Tenebrio molitor [Coleoptera: Tenebrionidae] 16 Oct. 12 ARG: San Juan, Pocito Experimental INTA [YPSS, SMYA $1 \%]\left[\mathrm{F}-20^{\circ} \mathrm{C}\right]$

CEP 396 J. Aguilera (C.C. López Lastra) Tenebrio molitor [Coleoptera: Tenebrionidae] 16 Oct. 12 ARG: San Juan, Finca Miguel Mas [YPSS, SMYA $1 \%]\left[\mathrm{F}-20^{\circ} \mathrm{C}\right]$

CEP 397 J. Aguilera (C.C. López Lastra) Tenebrio molitor [Coleoptera: Tenebrionidae] 25 Oct. 12 ARG: San Juan, Finca Miguel Mas [YPSS, SMYA $1 \%]$ [F-20 $\left.0^{\circ} \mathrm{C}\right]$

CEP 398 J. Aguilera (C.C. López Lastra) Tenebrio molitor [Coleoptera: Tenebrionidae] 25 Oct. 12 ARG: San Juan, Pocito Experimental, INTA [YPSS, SMYA $1 \%]\left[\mathrm{F}-20^{\circ} \mathrm{C}\right]$

CEP 399 J. Aguilera (C.C. López Lastra) Tenebrio molitor [Coleoptera: Tenebrionidae] 25 Oct. 12 ARG: San Juan, Pocito, Experimental INTA [YPSS, SMYA $1 \%$ ] [F-20 $\left.{ }^{\circ} \mathrm{C}\right]$

CEP 400 J. Aguilera (C.C. López Lastra) Tenebrio molitor [Coleoptera: Tenebrionidae] 25 Oct. 12 ARG: San Juan, Pocito
Experimental INTA [YPSS, SMYA 1\%] [F-20 $\left.{ }^{\circ} \mathrm{C}\right]$

CEP 401 J. Aguilera (C.C. López Lastra) Tenebrio molitor [Coleoptera: Tenebrionidae] 3 Nov. 12 ARG: San Juan, Pocito Experimental INTA [YPSS, SMYA $1 \%]\left[\mathrm{F}-20^{\circ} \mathrm{C}\right]$

CEP 403 J. Aguilera (C.C. López Lastra) Tenebrio molitor [Coleoptera: Tenebrionidae] 3 Nov. 12 ARG: San Juan, Finca Miguel Mas [YPSS, SMYA 1\%] [P]

CEP 404 J. Aguilera (C.C. López Lastra) Tenebrio molitor [Coleoptera: Tenebrionidae] 3 Nov. 12 ARG: San Juan, Pocito Experimental INTA [YPSS, SMYA $1 \%]\left[\mathrm{F}-20^{\circ} \mathrm{C}, \mathrm{F}-70^{\circ} \mathrm{C}, \mathrm{P}, \mathrm{AD}\right]$ CEP 405 J. Aguilera (C.C. López Lastra) Tenebrio molitor [Coleoptera: Tenebrionidae] 3 Nov. 12 ARG: San Juan, Pocito Experimental INTA [YPSS, SMYA $1 \%]\left[\mathrm{F}-20^{\circ} \mathrm{C}, \mathrm{F}-70^{\circ} \mathrm{C}, \mathrm{L}\right]$

CEP 406 J. Aguilera (C.C. López Lastra) Tenebrio molitor [Coleoptera: Tenebrionidae] 3 Nov. 12 ARG: San Juan, Pocito Experimental INTA [YPSS, SMYA 1\%] [P]

CEP 407 J. Aguilera (C.C. López Lastra) Tenebrio molitor [Coleoptera: Tenebrionidae] 3 Nov. 12 ARG: San Juan, Finca Tía Nora [YPSS, SMYA 1\%] [P]

CEP 408 J. Aguilera (C.C. López Lastra) Tenebrio molitor [Coleoptera: Tenebrionidae] 18 Nov. 12 ARG: San Juan, Finca Miguel Mas [YPSS, SMYA 1\%] [P]

CEP 409 J. Aguilera (C.C. López Lastra) Tenebrio molitor [Coleoptera: Tenebrionidae] 18 Nov. 12 ARG: San Juan, Pocito Experimental INTA [YPSS, SMYA $1 \%$ ] [F- $\left.20^{\circ} \mathrm{C}, \mathrm{P}, \mathrm{AD}\right]$ CEP 410 J. Aguilera (C.C. López Lastra) Tenebrio molitor [Coleoptera: Tenebrionidae] 18 Nov. 12 ARG: San Juan, Pocito Experimental INTA [YPSS, SMYA $1 \%$ ] [F-20 ${ }^{\circ} \mathrm{C}, \mathrm{P}, \mathrm{AD}$ ] CEP 411 J. Aguilera (C.C. López Lastra) Tenebrio molitor [Coleoptera: Tenebrionidae] 18 Nov. 12 ARG: San Juan, Finca Miguel Mas [YPSS, SMYA $1 \%$ ] [F- $20^{\circ} \mathrm{C}, \mathrm{F}-70^{\circ} \mathrm{C}, \mathrm{P}$ ]

CEP 412 J. Aguilera (C.C. López Lastra) Tenebrio molitor [Coleoptera: Tenebrionidae] 18 Nov. 12 ARG: San Juan, Finca Miguel Mas [YPSS, SMYA 1\%] [P, AD]

CEP 413 J. Aguilera (C.C. López Lastra) Tenebrio molitor [Coleoptera: Tenebrionidae] 18 Nov. 12 ARG: San Juan, Finca Miguel Mas [YPSS, SMYA $1 \%$ ] [F- $20^{\circ} \mathrm{C}, \mathrm{F}-70^{\circ} \mathrm{C}, \mathrm{P}$ ]

CEP 416 J. Aguilera (C.C. López Lastra) Tenebrio molitor [Coleoptera: Tenebrionidae] 15 Jun. 13 ARG: Entre Ríos, Colón, P. N. El Palmar [YPSS, SMYA 1\%] [F- $\left.70^{\circ} \mathrm{C}\right]$

CEP 423 C.C. López Lastra (C.C. López Lastra) Tenebrio molitor [Coleoptera: Tenebrionidae] 15 Mar. 13 ARG: Bs. As., La Plata, Tolosa [YPSS, SMYA 1\%] [L]

CEP 434 C.C. López Lastra (C.C. López Lastra) Tenebrio molitor [Coleoptera: Tenebrionidae] 17 Jul. 14 ARG: Bs. As., La Plata, Los Hornos, estación experimental de arroz [YPSS, SMYA 1\%] $\left[\mathrm{F}-20^{\circ} \mathrm{C}, \mathrm{F}-20^{\circ} \mathrm{C}, \mathrm{AD}, \mathrm{L}\right]$

CEP 435 (C.C. López Lastra) Tenebrio molitor [Coleoptera: Tenebrionidae] 17 Jul. 14 ARG: Bs. As., La Plata, Los Hornos, est. exp. de arroz [YPSS, SMYA $1 \%$ ] [F- $\left.20^{\circ} \mathrm{C}, \mathrm{F}-20^{\circ} \mathrm{C}, \mathrm{AD}, \mathrm{L}\right]$ 
CEP 542 (C.C. López Lastra) Tenebrio molitor [Coleoptera: Tenebrionidae] suelo de cultivo de Fragaria sp., 22 Jun. 14 ARG: Bs. As., La Plata, Los Hornos [SMYA 1/4] [F-20 ${ }^{\circ} \mathrm{C}, \mathrm{P}, \mathrm{AD}$ ] CEP 543 (C.C. López Lastra) Tenebrio molitor [Coleoptera: Tenebrionidae] 22 Jun. 14 ARG: Bs. As., La Plata, Los Hornos [SMYA $1 / 4$ ] [F-20 $\left.{ }^{\circ} \mathrm{C}, \mathrm{P}, \mathrm{AD}\right]$

CEP 544 F. Achinelly (C.C. López Lastra) Tenebrio molitor [Coleoptera: Tenebrionidae] 22 Jun. 14 ARG: Bs. As., La Plata, Los Hornos [SMYA 1/4] [F-20 $\mathrm{C}, \mathrm{P}, \mathrm{AD}$ ]

CEP 559 A. Salas (J. Tornesello) Tenebrio molitor [Coleoptera: Tenebrionidae] Ago. 15 ARG: Bs. As., La Plata, Cnia. Urquiza, Los Hornos [SMYA 1/4] [AD, L]

CEP 581 N. Borrelli (C.C. López Lastra) Tenebrio molitor [Coleoptera: Tenebrionidae] 19 Jul. 16 ARG: Bs. As., P. Pereyra [SDYA 1/4] [F -20 y F-70 ${ }^{\circ} \mathrm{C}, \mathrm{P}, \mathrm{AD}$ ]

CEP 582 N. Borrelli (C.C. López Lastra) Tenebrio molitor [Coleoptera: Tenebrionidae], 19 Jul. 16 ARG: Bs. As., P. Pereyra [SDYA 1/4] [F -20, F-70 ${ }^{\circ} \mathrm{C}, \mathrm{P}, \mathrm{AD}$ ]

CEP $583 \mathrm{~N}$. Borrelli (C.C. López Lastra) Tenebrio molitor [Coleoptera: Tenebrionidae] suelo ganadero, 19 Jul. 16 ARG: Entre Rios [SDYA 1/4] [F - 20, F-70 $\left.{ }^{\circ} \mathrm{C}, \mathrm{P}, \mathrm{AD}\right]$

CEP 584 E. Ordoqui (C.C. López Lastra) Tenebrio molitor [Coleoptera: Tenebrionidae] suelo orgánico, 22 Jul. 16 ARG: Bs. As. P. Pereyra [SDYA 1/4] [F - 20, F-70 ${ }^{\circ} \mathrm{C}, \mathrm{AD}$ ]

CEP 585 E. Ordoqui (C.C. López Lastra) Tenebrio molitor [Coleoptera: Tenebrionidae] suelo orgánico, 22 Jul. 16 ARG: Bs. As. P. Pereyra [SDYA 1/4] [F - 20, F-70 ${ }^{\circ} \mathrm{C}, \mathrm{P}, \mathrm{AD}$ ]

CEP 586 F. Bozza (C.C. López Lastra) Tenebrio molitor [Coleoptera: Tenebrionidae] suelo orgánico, 22 Jul. 16 ARG: Bs. As. P. Pereyra [SDYA 1/4] [F -20, F-70 $\left.{ }^{\circ} \mathrm{C}, \mathrm{P}, \mathrm{AD}\right]$

CEP 587 F. Bozza (C.C. López Lastra) Tenebrio molitor [Coleoptera: Tenebrionidae] suelo orgánico 22 Jul. 16 ARG: Bs. As. P. Pereyra [SDYA 1/4] [F-70 $\left.{ }^{\circ} \mathrm{C}, \mathrm{P}, \mathrm{AD}, \mathrm{L}\right]$

CEP 589 J. Aguilera (J. Aguilera) Tenebrio molitor [Coleoptera: Tenebrionidae] suelo, 10 Mar. 16. ARG, San Juan, Pocito. [SDYA 1/4] [F-70 ${ }^{\circ} \mathrm{C}, \mathrm{P}, \mathrm{AD}, \mathrm{L}$ ]

CEP 590 J. Aguilera (J. Aguilera) Tenebrio molitor [Coleoptera: Tenebrionidae] suelo, 10 Mar. 16. ARG, San Juan, Pocito. [SDYA 1/4] [F-70 ${ }^{\circ} \mathrm{C}, \mathrm{P}, \mathrm{AD}, \mathrm{L}$ ]

CEP 591 J. Aguilera (J. Aguilera) Tenebrio molitor [Coleoptera: Tenebrionidae] suelo 10 Mar. 16. ARG, San Juan, Pocito. [SDYA 1/4] [F-70 $\left.{ }^{\circ} \mathrm{C}, \mathrm{P}, \mathrm{AD}, \mathrm{L}\right]$

CEP 592 M. Lattari (C.C. López Lastra) Tenebrio molitor [Coleoptera: Tenebrionidae] suelo, 27 Oct. 16 ARG: Salta, Ruta Nac. 9 km 1643 [SDYA 1/4] [F-20 y F $-70^{\circ} \mathrm{C}, \mathrm{L}$ ] CEP 593 M. Lattari (C.C. López Lastra) Tenebrio molitor [Coleoptera: Tenebrionidae] suelo, 27 Oct. 16 ARG: Salta, Ruta Nac. 9 km 1643 [SDYA 1/4] [F-20 y F-70 ${ }^{\circ} \mathrm{C}, \mathrm{L}$ ] CEP 594 M. Lattari (C.C. López Lastra) Tenebrio molitor [Coleoptera: Tenebrionidae] suelo, 27 Oct. 16 ARG: Salta,
Ruta Nac. 9 km 1643 [SDYA 1/4] [F-20 y F-70 ${ }^{\circ} \mathrm{C}, \mathrm{L}$ ] CEP 595 M. Lattari (C.C. López Lastra) Tenebrio molitor [Coleoptera: Tenebrionidae] suelo, 27 Oct. 16 ARG: Salta, Ruta Nac. 9 km 1643 [SDYA 1/4] [F-20 y F-70 ${ }^{\circ} \mathrm{C}$ ] CEP 596 E. Ordoqui (R. Manfrino) Tenebrio molitor [Coleoptera: Tenebrionidae] suelo, 13 Jul. 16 ARG: Bs. As, P. Pereyra [SDYA 1/4] [F-20, F-70 $\left.{ }^{\circ} \mathrm{C}, \mathrm{P}, \mathrm{L}\right]$

CEP 597 E. Ordoqui (R. Manfrino) Tenebrio molitor [Coleoptera: Tenebrionidae] suelo, 13 Jul. 16 ARG: Bs. As, P. Pereyra [SDYA 1/4] [F-20, F-70 $\left.{ }^{\circ} \mathrm{C}, \mathrm{L}\right]$

CEP 598 E. Ordoqui (R. Manfrino) Tenebrio molitor [Coleoptera: Tenebrionidae] suelo, 13 Jul. 16 ARG: Bs. As, P. Pereyra [SDYA 1/4] [F-20, F-70 $\left.{ }^{\circ} \mathrm{C}, \mathrm{L}\right]$

CEP 599 E. Ordoqui (R. Manfrino) Tenebrio molitor [Coleoptera: Tenebrionidae] suelo, 13 Jul. 16 ARG: Bs. As, P. Pereyra [SDYA 1/4] [F-20, F-70 $\left.{ }^{\circ} \mathrm{C}, \mathrm{L}\right]$

CEP 600 E. Ordoqui (R. Manfrino) Tenebrio molitor [Coleoptera: Tenebrionidae] suelo, 13 Jul. 16 ARG: Bs. As, P. Pereyra [SDYA 1/4] [F-20, F-70 $\left.{ }^{\circ} \mathrm{C}, \mathrm{L}\right]$

CEP 615 A. Musso (C.C. López Lastra) 13 Ago. 16 Tenebrio molitor [Coleoptera: Tenebrionidae] ARG: Bs. As. Lincoln, Martinez de Hoz [SDYA 1/4] [F-70C ${ }^{\circ}, \mathrm{P}, \mathrm{L}$ ]

CEP 616 A. Musso (C.C. López Lastra) 13 Ago. 16 Tenebrio molitor [Coleoptera: Tenebrionidae] ARG: Bs. As. Lincoln, Martinez de Hoz [SDYA 1/4] [F-70C ${ }^{\circ}, \mathrm{P}, \mathrm{L}$ ]

CEP 617 A. Musso C.C. López Lastra) 13 Ago. 16 Tenebrio molitor [Coleoptera: Tenebrionidae] ARG: Bs. As. Lincoln, Martinez de Hoz [SDYA 1/4] [F-70C ${ }^{\circ}$, L]

CEP 618 A. Musso (C.C. López Lastra) 15 Ago. 16 Tenebrio molitor [Coleoptera: Tenebrionidae] suelo quinta orgánica, ARG: Bs. As., Berazategui, P. Pereyra [SDYA 1/4] [F-70C ${ }^{\circ}, \mathrm{P}, \mathrm{L}$ ] CEP 619 A. Musso (C.C. López Lastra) 15 Ago. 16 Tenebrio molitor [Coleoptera: Tenebrionidae] suelo de quinta orgánica ARG: Bs. As., Berazategui, P. Pereyra [SDYA 1/4] [F-70C ${ }^{\circ}$, P] CEP 620 A. Musso (C.C. López Lastra) 15 Ago. 16 Tenebrio molitor [Coleoptera: Tenebrionidae] suelo de quinta orgánica ARG: Bs. As., Berazategui, P. Pereyra [SDYA 1/4] [F-70C ${ }^{\circ}, \mathrm{P}, \mathrm{L}$ ] CEP 621 A. Musso (C.C. López Lastra) 15 Ago. 16 Tenebrio molitor [Coleoptera: Tenebrionidae] suelo de quinta orgánica, ARG: Bs. As., Berazategui, P. Pereyra [SDYA 1/4] [F-70C ${ }^{\circ}, \mathrm{P}, \mathrm{L}$ ] CEP 622 A. Musso (C.C. López Lastra) 15 Ago. 16 Tenebrio molitor [Coleoptera: Tenebrionidae] suelo de quinta orgánica, ARG: Bs. As., Berazategui, P. Pereyra [SDYA 1/4] [F-70C ${ }^{\circ}, \mathrm{P}, \mathrm{L}$ ] CEP 623 A. Musso (C.C. López Lastra) 15 Ago. 16 Tenebrio molitor [Coleoptera: Tenebrionidae] suelo orgánico, ARG: Bs. As., Berazategui, P. Pereyra [SDYA 1/4] [F-70C $\left.{ }^{\circ}, \mathrm{P}, \mathrm{L}\right]$ CEP 615 A. Musso (C.C. López Lastra) Suelo cebo Tenebrio molitor [Coleoptera: Tenebrionidae] 13 Ago. 16 ARG: Buenos Aires, Pdo. Lincoln, Martínez de Hoz [SDYA 1/4] [F-70 ${ }^{\circ} \mathrm{C}, \mathrm{P}, \mathrm{L}$ ] CEP 616 A. Musso (C.C. López Lastra) Suelo cebo Tenebrio 
molitor [Coleoptera: Tenebrionidae] 13 Ago. 16 ARG: Buenos Aires, Pdo. Lincoln, Martínez de Hoz [SDYA 1/4] [F-70 ${ }^{\circ} \mathrm{C}, \mathrm{P}, \mathrm{L}$ ] CEP 617 A. Musso (C.C. López Lastra) Suelo cebo Tenebrio molitor [Coleoptera: Tenebrionidae] 13 Ago. 16 ARG: Buenos Aires, Pdo. Lincoln, Martínez de $\mathrm{Hoz}$ [SDYA 1/4] [F- $70^{\circ} \mathrm{C}, \mathrm{P}, \mathrm{L}$ ] CEP 618 E. Ordoqui \& F. Boza (R. Manfrino) Suelo orgánico Tenebrio molitor Coleoptera: Tenebrionidae] 15 Ago. 16 ARG: Buenos Aires, Pque. Pereyra Iraola [SDYA $1 / 4]\left[\mathrm{F}-70^{\circ} \mathrm{C}, \mathrm{P}, \mathrm{L}\right]$ CEP 619 E. Ordoqui \& F. Boza (R. Manfrino) Suelo orgánico Tenebrio molitor [Coleoptera: Tenebrionidae] 15 Ago. 16 ARG: Buenos Aires, Pque. Pereyra Iraola [SDYA $1 / 4]\left[\mathrm{F}-70^{\circ} \mathrm{C}, \mathrm{P}, \mathrm{L}\right]$ CEP 620 E. Ordoqui \& F. Boza (R. Manfrino) 15 Ago. 16 Suelo orgánico Tenebrio molitor [Coleoptera: Tenebrionidae] ARG: Buenos Aires, Pque. Pereyra Iraola [SDYA 1/4] [F-70 $\left.{ }^{\circ} \mathrm{C}, \mathrm{P}, \mathrm{L}\right]$ CEP 621 E. Ordoqui \& F. Boza (R. Manfrino) 15 Ago. 16 Suelo orgánico Tenebrio molitor [Coleoptera: Tenebrionidae] ARG: Buenos Aires, Pque. Pereyra Iraola [SDYA 1/4] [F-70 $\left.{ }^{\circ} \mathrm{C}, \mathrm{P}, \mathrm{L}\right]$ CEP 622 E. Ordoqui \& F. Boza (R. Manfrino) Suelo orgánico Tenebrio molitor 15 Ago. 16 [Coleoptera: Tenebrionidae] ARG: Buenos Aires, Pque. Pereyra Iraola [SDYA $1 / 4]\left[\mathrm{F}-70^{\circ} \mathrm{C}, \mathrm{P}, \mathrm{L}\right]$ CEP 623 E. Ordoqui \& F. Boza (R. Manfrino) Suelo orgánico Tenebrio molitor [Coleoptera: Tenebrionidae] 15 Ago. 16 ARG: Buenos Aires, Pque. Pereyra Iraola [SDYA $1 / 4]\left[\mathrm{F}-70^{\circ} \mathrm{C}, \mathrm{P}, \mathrm{L}\right]$ CEP 624 E. Ordoqui \& F. Boza (R. Manfrino) Suelo organico Tenebrio molitor [Coleoptera: Tenebrionidae] 15 Ago. 16 ARG: Buenos Aires, Pque. Pereyra Iraola [SDYA $1 / 4]\left[\mathrm{F}-70^{\circ} \mathrm{C}, \mathrm{P}, \mathrm{L}\right]$ CEP 625 E. Ordoqui \& F. Boza (R. Manfrino) Suelo orgánico Tenebrio molitor [Coleoptera: Tenebrionidae] 15 Ago. 16 ARG: Buenos Aires, Pque. Pereyra Iraola [SDYA 1/4] [F-70 $\left.{ }^{\circ} \mathrm{C}, \mathrm{P}, \mathrm{L}\right]$ CEP 626 E. Ordoqui \& F. Boza (R. Manfrino) Suelo orgánico Tenebrio molitor [Coleoptera: Tenebrionidae] 15 Ago. 16 ARG: Buenos Aires, Pque. Pereyra Iraola [SDYA 1/4] [F-70 $\left.{ }^{\circ} \mathrm{C}, \mathrm{P}, \mathrm{L}\right]$ CEP679 R. Manfrino (R. Manfrino) Tenebrio molitor [Coleoptera: Tenebrionidae] suelo monte de ciruela, 15 Jul. 17 ARG: Bs As. Est. Exp. Los Hornos, FCAyF [SDYA $1 / 4]$ [ F- $\left.70^{\circ} \mathrm{C}, \mathrm{P}, \mathrm{L}\right]$ CEP680 R. Manfrino (R. Manfrino) Tenebrio molitor [Coleoptera: Tenebrionidae] suelo monte de ciruela, 15 Jul. 17 ARG: Bs As. Est. Exp. Los Hornos, FCAyF [SDYA 1/4] [F-70 $\left.{ }^{\circ} \mathrm{C}, \mathrm{P}, \mathrm{L}\right]$ CEP 681 R. Manfrino (R. Manfrino) Tenebrio molitor [Coleoptera: Tenebrionidae] suelo monte de nogal, 15 Jul. 17 ARG: Bs As. Est. Exp. Los Hornos, FCAyF [SDYA 1/4] [F-70 $\left.{ }^{\circ} \mathrm{C}, \mathrm{P}, \mathrm{L}\right]$

CEP 682 R. Manfrino (R. Manfrino) Tenebrio molitor [Coleoptera: Tenebrionidae] suelo monte de durazno, 15 Jul. 17 ARG: Bs As. Est. Exp. Los Hornos, FCAyF [SDYA 1/4] [F-70 $\left.{ }^{\circ} \mathrm{C}, \mathrm{P}, \mathrm{L}\right]$ CEP 683 A. Musso (A. Musso) Tenebrio molitor [Coleoptera: Tenebrionidae] suelo, 18 Jul. 17 ARG: Bs As. Est. Exp. Los Hornos, FCAyF [SDYA $1 / 4]$ [F-70 $\left.{ }^{\circ} \mathrm{C}, \mathrm{P}, \mathrm{L}\right]$

CEP 684 A. Musso (A. Musso) Tenebrio molitor [Coleoptera: Tenebrionidae] suelo, 18 Jul. 17 ARG: Bs As. Est. Exp. Los Hornos, FCAyF [SDYA $1 / 4]$ [F-70 $\left.{ }^{\circ} \mathrm{C}, \mathrm{P}, \mathrm{L}\right]$

\section{Metarhizium brunneum Petch}

CEP 005 [ARSEF 2974] López Lastra, C.C. (López Lastra, C.C.) [Diptera: Culicidae] Ocheloratus crinifer ARG Buenos Aires, Berisso, Los Talas. [SDYA] [F-70 $\left.{ }^{\circ} \mathrm{C}, \mathrm{P}, \mathrm{L}\right] .\left(^{*}\right)\left(^{* *}\right)$

CEP 578 [ARSEF 6120] (C.C. López Lastra) Diloboderus abderus larva suelo, 3 Jun. 16 ARG: Buenos Aires, Lincoln. [SDYA 1/4] [F-20, F-70C, P, AD] $\left({ }^{*}\right)\left({ }^{* *}\right)$

Metarhizium rileyi (Farlow) Kepler, Rehner \& Humber CEP 004 [ARSEF 5205; LPS 408] C.C. López Lastra, Rachiplusia nu [Lepidoptera: Noctuidae] 1 Mar. 94 ARG: Buenos Aires, Los Hornos, [SMYA 1\%] [F-20 ${ }^{\circ} \mathrm{C} \mathrm{P}$ ]

CEP 021 [ARSEF 7479] V. Dikgolz, (V. Dikgolz \& C.C. López Lastra), Anticarsia gemmatalis [Lepidoptera: Noctuidae] suelo cultivado con Glycine max, 1 Abr. 01 ARG: Buenos Aires, Chivilcoy [YPSS, SMYA 1\%] [F-20 $\left.0^{\circ} \mathrm{C}, \mathrm{P}, \mathrm{AD}\right] \mathrm{B} . \mathrm{b}$

CEP 040 [ARSEF 7056] V. Dikgolz (V. Dikgolz), Anticarsia gemmatalis [Lepidoptera: Noctuidae], 1 May. 02 ARG: Buenos Aires, La Plata [YPSS, SMYA 1/4] [F- $\left.20^{\circ} \mathrm{C}, \mathrm{F}-70^{\circ} \mathrm{C} \mathrm{P}, \mathrm{AD}\right]$ CEP 065 [ARSEF 7052] V. Dikgolz (C.C. López Lastra), Anticarsia gemmatalis [Lepidoptera: Noctuidae] 15 May. 01 ARG: Buenos Aires, Magdalena, General Mansilla [YPSS, SMYA 1\%] [F-20 ${ }^{\circ} \mathrm{C}, \mathrm{F}-70^{\circ} \mathrm{C}, \mathrm{P}, \mathrm{AD}$ ]

CEP 367 R. Manfrino (R. Manfrino \& C.C. López Lastra), Plutella xylostella [Lepidoptera: Plutellidae] 29 Jul. 12 ARG: Buenos Aires, La Plata, [YPSS, SMYA $1 \%]\left[\mathrm{F}-70^{\circ} \mathrm{C}, \mathrm{L}\right]\left({ }^{*}\right)\left({ }^{* *}\right)$ CEP 422 C.C. López Lastra (C.C. López Lastra \& V. Dikgolz), Anticarsia gemmatalis [Lepidoptera: Noctuidae] suelo con Glycine max, 20 May. 02 ARG: Buenos Aires, Zavalla [SEMA] $\left[\mathrm{F}-20^{\circ} \mathrm{C}, \mathrm{F}-70^{\circ} \mathrm{C} \mathrm{L}\right]\left({ }^{*}\right)\left({ }^{* *}\right)$

\section{Mucor sp.}

CEP 489 P. Albornoz Medina (M. Cabello) Anastrepha fraterculus (Diptera: Tephritidae) ARG: Tucumán, Horco Molle, Yerba Buena 27 Mar 09. [SDYA 1/4] [F-20 ${ }^{\circ} \mathrm{C}$ ]

CEP 504 P. Albornoz Medina (M. Cabello) Anastrepha fraterculus (Diptera: Tephritidae) ARG: Tucumán, Horco Molle, Yerba Buena 08 Abr 09. [SDYA 1/4] F-20 ${ }^{\circ} \mathrm{C}$ ]

CEP 513 P. Albornoz Medina (M. Cabello) Anastrepha sp. (Diptera: Tephritidae) ARG: Tucumán, Horco Molle, Yerba Buena 20 Ene 10. [SDYA 1/4] [F-20 ${ }^{\circ} \mathrm{C}$ ]

CEP 515 P. Albornoz Medina (M. Cabello) Anastrepha fraterculus (Diptera: Tephritidae) ARG: Tucumán, Horco Molle, Yerba Buena 26 Ene 10. [SDYA 1/4] [F-20 ${ }^{\circ} \mathrm{C}$ ]

[AD]

Nigelia martiale (Speg.) Luangsa-ard \& Thanakitp ( $\mathrm{Ex}=$ Metacordyceps martialis)

605 B. Moreno (C.C. López Lastra) Cerambycidae NI [Coleoptera: Cerambycidae] 18 Nov. 16. ARG: Misiones. P.P. Península [SDYA 1/4] [F-70 $\left.{ }^{\circ} \mathrm{C}, \mathrm{P}, \mathrm{L}\right]$ 


\section{Penicillium sp.}

CEP 519 P. Albornoz (M. Cabello) Anastrepha sp (Diptera: Tephritidae) ARG: Tucumán, Horco Molle, Yerba Buena 26 Ene 10. [SDYA 1/4] [F-20 $\left.0^{\circ} \mathrm{C}\right][\mathrm{AD}]\left[\mathrm{F}-20^{\circ} \mathrm{C}\right]$

CEP 520 P. Albornoz (M. Cabello) P. Albornoz (M. Cabello) Anastrepha sp (Diptera: Tephritidae) ARG: Tucumán, Horco Molle, Yerba Buena 26 Ene 10. [SDYA 1/4] [F-20 $\left.0^{\circ} \mathrm{C}\right]$

CEP 522 P. Albornoz (M. Cabello) P. Albornoz (M. Cabello) Ceratitis capitata (Diptera: Tephritidae) ARG: Tucumán, Horco Molle, Yerba Buena $15 \mathrm{Abr}$. 10. [SDYA 1/4] [F-20 ${ }^{\circ} \mathrm{C}$ ]

CEP 525 P. Albornoz (M. Cabello) [Anastrepha fraterculus (Diptera: Tephritidae) ARG: Tucumán, Horco Molle, Yerba Buena 26 Ene 10. [SDYA 1/4] [F-20 $\left.{ }^{\circ} \mathrm{C}\right]$

CEP 526 P. Albornoz (M. Cabello) Anastrepha fraterculus (Diptera: Tephritidae) ARG: Tucumán, Horco Molle, Yerba Buena 26 Ene 10. [SDYA 1/4] [F-20 $\left.{ }^{\circ} \mathrm{C}\right]$

CEP 530 P. Albornoz (M. Cabello) Anastrepha fraterculus (Diptera: Tephritidae) ARG: Tucumán, Horco Molle, Yerba Buena 05 Ene 10. [SDYA 1/4] [F-20 $\left.{ }^{\circ} \mathrm{C}\right]$.

Purpureocillium lilacinum (Thom) Luangsa-ard, Houbraken, Hywel-Jones \& Samson

CEP 071 [ARSEF 7482] G. Marti (López Lastra) Triatoma infestans [Heteroptera: Reduviidae] 17 Dic. 03 ARG: Dto. Giménez, El Quebracho, peridomicilio [AM, SDYA] [AD] (*) CEP 349 M.E. Schapovaloff (M.E. Schapovaloff \& C.C. López Lastra), suelo cultivado con Ilex paraguariensis 17 Feb. 09 ARG: Misiones. Santa Inés [AM, SDYA] [F- $20^{\circ} \mathrm{C}, \mathrm{F}-20^{\circ} \mathrm{C}, \mathrm{P}, \mathrm{AD}$ ( $\left(^{*}\right)$ CEP 351 M.E. Schapovaloff (M.E. Schapovaloff \& C.C. López Lastra), suelo cultivado con llex paraguariensis 2 Jun. 09 ARG: Misiones, Jardín América [AM, SDYA] [F-20 $\left.2{ }^{\circ} \mathrm{C}, \mathrm{AD}\right]\left({ }^{*}\right)$ CEP 352 M.E. Schapovaloff (M.E. Schapovaloff \& C.C. López Lastra, suelo cultivado con llex paraguariensis 2 Jun 09 ARG: Misiones, Apóstoles [AM, SDYA] [F-20 $\left.0^{\circ} \mathrm{C}, \mathrm{P}, \mathrm{AD}\right]\left({ }^{*}\right)$ CEP 354 M.E. Schapovaloff (M.E. Schapovaloff \& C.C. López Lastra), suelo cultivado con Ilex paraguariensis 25 Jun. 09 ARG: Misiones, Apóstoles [AM, SDYA] [F-20 $\left.2{ }^{\circ} \mathrm{C}, \mathrm{AD}\right]\left({ }^{*}\right)$ CEP 355 M.E. Schapovaloff (M.E. Schapovaloff \& C.C. López Lastra, suelo cultivado con llex paraguariensis 30 Jun. 09 ARG: Misiones, Apóstoles [AM, SDYA] [F- $\left.20^{\circ} \mathrm{C}, \mathrm{P}, \mathrm{AD}\right]\left(^{*}\right)$ CEP 356 M.E. Schapovaloff (M.E. Schapovaloff \& C.C. López Lastra) suelo cultivado con llex paraguariensis 17 Feb. 09 ARG: Misiones, Santa Inés [AM, SDYA] [F-20 $\left.{ }^{\circ} \mathrm{C}, \mathrm{AD}\right]\left({ }^{*}\right)$ CEP 550 L. Elizalde (C.C. López Lastra) Acromyrmex lobicornis [Hymenoptera: Formicidae] 28 Mar. 14 ARG: R. Negro Villa Llanquín, La Lipela [AM, SDYA] [F-20 $\left.{ }^{\circ} \mathrm{C}, \mathrm{P}, \mathrm{AD}, \mathrm{L}\right]\left(^{*}\right)$

CEP 555 J. Barneche (C.C. López Lastra), Stenoterommata platensis [Aranea: Nemesidae] 20 Jun. 14 ARG: Buenos Aires, Magdalena, R. P. Pearson [AM, SDYA] [F- $20^{\circ} \mathrm{C}, \mathrm{F}-70^{\circ} \mathrm{C} P, A D$, L] $(*)$
CEP 643 B. Moreno (C.C. López Lastra) [Hemiptera: Cicadidae] 31 Ene. 17 ARG: Juj., P.N. Calilegua [SDYA 1/4] [F-70 ${ }^{\circ} \mathrm{C}, \mathrm{P}, \mathrm{L}$ ] CEP 646 B. Moreno (C.C. López Lastra) [Hemiptera: Pentatomidae] 8 Abr. 17 ARG: Misiones, P.P. Península [SDYA 1/4] [F-70 $\mathrm{C}, \mathrm{P}, \mathrm{L}]$

CEP 648 B. Moreno (C.C. López Lastra) [Lepidoptera] 8 Abr. 17 ARG: Misiones, P.P. Península [SDYA 1/4] [F-70 $\left.{ }^{\circ} \mathrm{C}, \mathrm{P}, \mathrm{L}\right]$ ( $\left.^{*}\right)$ CEP 649 B. Moreno (C.C. López Lastra) [Lepidoptera] 8 Abr. 17 ARG: Misiones, P.P. Península [SDYA 1/4] [F-70 $\left.{ }^{\circ} \mathrm{C}, \mathrm{P}, \mathrm{L}\right]$

CEP 652 B. Moreno (C.C. López Lastra) [Hemiptera: Pentatomidae] 9 Abr. 17 ARG: Misiones, P.P. Península [SDYA $1 / 4]\left[\mathrm{F}-70^{\circ} \mathrm{C}, \mathrm{P}, \mathrm{L}\right]$

CEP 655 B. Moreno (C.C. López Lastra) [Hemiptera: Pentatomidae] 9 Abr. 17 ARG: Misiones, P.P. Península [SDYA 1/4] [F-70 $\mathrm{C}, \mathrm{P}, \mathrm{L}]$

CEP 657 B. Moreno (C.C. López Lastra) [Hemiptera: Pentatomidae] 9 Abr. 17 ARG: Misiones, P.P. Península [SDYA $1 / 4]\left[F-70^{\circ} \mathrm{C}, \mathrm{P}, \mathrm{L}\right]$

CEP 658 B. Moreno (C.C. López Lastra) [Hemiptera: Pentatomidae] 9 Abr. 17 ARG: Misiones, P.P. Península [SDYA $1 / 4]\left[F-70^{\circ} \mathrm{C}, \mathrm{P}, \mathrm{L}\right]$

CEP 659 B. Moreno (C.C. López Lastra) [Hemiptera: Pentatomidae] 9 Abr. 17 ARG: Misiones, P.P. Península [SDYA $1 / 4]\left[F-70^{\circ} \mathrm{C}, \mathrm{P}, \mathrm{L}\right]$

CEP 660 B. Moreno (C.C. López Lastra) [Hemiptera: Pentatomidae] 9 Abr. 17 ARG: Misiones, P.P. Península [SDYA $1 / 4]\left[\mathrm{F}-70^{\circ} \mathrm{C}, \mathrm{P}, \mathrm{L}\right]$

CEP 661 B. Moreno (C.C. López Lastra) [Hemiptera: Pentatomidae] 9 Abr. 17 ARG: Misiones, P.P. Península [SDYA $1 / 4]\left[F-70^{\circ} \mathrm{C}, \mathrm{P}, \mathrm{L}\right]$

CEP 664 B. Moreno (C.C. López Lastra) [Hemiptera: Pentatomidae] 9 Abr. 17 ARG: Misiones, P.P. Península [SDYA $1 / 4]\left[\mathrm{F}-70^{\circ} \mathrm{C}, \mathrm{P}, \mathrm{L}\right]$

CEP 670 B. Moreno (C.C. López Lastra) [Hemiptera: Pentatomidae] 9 Abr. 17 ARG: Misiones, P.P. Península [SDYA $1 / 4]\left[\mathrm{F}-70^{\circ} \mathrm{C}, \mathrm{P}, \mathrm{L}\right]$

CEP 671 B. Moreno (C.C. López Lastra) [Hemiptera: Pentatomidae] 9 Abr. 17 ARG: Misiones, P.P. Península [SDYA $1 / 4]\left[\mathrm{F}-70^{\circ} \mathrm{C}, \mathrm{P}, \mathrm{L}\right]$

677 B. Moreno (C.C. López Lastra) [Hemiptera: Pentatomidae] 9 Abr. 17 ARG: Misiones, P.P. Península [SDYA 1/4] [F-70 ${ }^{\circ} \mathrm{C}, \mathrm{P}$, L]

\section{Staphylotrichum sp.}

CEP 281 G. Marti (G. Marti), Triatoma infestans [Heteroptera: Reduviidae] 17 Dic. 03 ARG: Santiago del Estero Dto. Giménez, El Quebracho, predomicilio [AM 2\%] [AD] (*)

\section{Talaromyces sp.}

CEP 272 G. Marti (G. Marti) Triatoma infestans [Heteroptera: Reduviidae] 17 Dic. 03 ARG: Santiago del Estero, Dto. 
Giménez, El Quebracho, predomicilio [AM, SDYA] [AD] $\left({ }^{*}\right)$

Zoophthora radicans (Bref.) A. Batko

CEP 030 [ARSEF 6917] A.C. Scorsetti (C.C. López Lastra), Tuta absoluta [Lepidoptera: Gelechiidae] Jun. 01 ARG: Buenos Aires, La Plata, Cnia. Urquiza [SDYA, SEMA] $\left[\mathrm{F}-70^{\circ} \mathrm{C}\right]\left({ }^{*}\right)$ CEP 173 [ARSEF 7789] C.C. López Lastra (C.C. López Lastra) Capitophorus eleagni [ Hemiptera: Aphididae) sobre Cynara cardunculus. 8 Sep. O4 ARG: Buenos Aires, La Plata, Cnia. Urquiza [SEMA] $\left[\mathrm{F}-70^{\circ} \mathrm{C}\right](*)$

CEP 320 [ARSEF 8466] A. Toledo (C.C. López Lastra) Epinotia aporema [Lepidoptera: Tortricidae] 13 May. 07 ARG: Buenos Aires, La Plata, Los Hornos [SEMA] [F-70 $\left.{ }^{\circ} \mathrm{C}\right]\left({ }^{*}\right)$

CEP 366. R. Manfrino (R. Manfrino \& C. C. López Lastra) Brevicoryne brassicae [Hemiptera: Aphididae] 27 May. 12 ARG: Monte Vera [AM, SDYA] [F-70 $\left.{ }^{\circ} \mathrm{C}\right]\left(^{*}\right)$

El listado total asciende a 684 taxones con 146 nuevas citas ampliando a 6 nuevas provincias, además de los datos ya publicados previos que corresponden a 12 en total y fueron 131 a partir de suelo con insectos "cebo" y 553 a partir de insectos. Los taxones de los artrópodos con respecto a insectos de los cuales fueron aislados las especies y cepas correspondieron a 9 ordenes: Coleoptera, Dermaptera, Diptera, Hemiptera, Hymenoptera, Orthoptera, Blattodea, Lepidoptera y algunos de Clase Arachnida, Orden Araneae. De las cepas registradas en este catálogo 107 han sido publicadas en artículos y de esas en 50 se han identificado por taxonomía molecular (genes ITS1, 5.8S, ITS2, además de las 95 secuenciadas por el proyecto IBOL ya citado).

\section{DISCUSIÓN}

Desde el área de la fitopatología existen numerosas técnicas y procedimientos para llegar a la identificación de los hongos fitopatógenos, si bien ellos describen los aislamientos en medios de cultivo, ya que son los que se recomiendan la mayoría de las veces puesto que son procedimientos sencillos (Godinez Rodriguez, 1996). Marcenaro Rodriguez (2013) cita en su catálogo que un amplio rango de aislamientos fueron identificados a partir de semillas de frijoles de diferentes regiones en Nicaragua. En el catálogo de ARSEF (2014) y el de EMBRAPA (2002) de EEUU y de Brasil respectivamente, se citaron las especies y aislamientos de hongos entomopatógenos incluyendo los datos específicos de recolección y de la identidad de sus hospedantes, siendo de las pocas colecciones y catálogos publicados que son exclusivos de insectos, al igual que el presente trabajo. En muchos otros artículos y publicaciones relacionadas sobre hongos, en lo que más se enfatiza es en su uso aplicado y se han editado más similares a los atlas que a catálogos. Este catálogo es un listado detallado que contribuye a ampliar el registro de especies y de cepas fúngicas en cultivo, con sus datos de ubicación geográfica, fechas de recolección, ambientes y hospedantes o sustratos.

\section{AGRADECIMIENTOS}

A la UNLP y fondos IBOL por el financiamiento parcial. Al Dr. Richard Humber y a la Dra. Louela Castrillo (ARSEF). A la Srta. Tornesello, por la preservación de los cultivos.

\section{REFERENCIAS}

ARSEF Catálogo. 2014. USDA-ARS Biological Integrated Pest Management Research. Ithaca, NewYork, USA. URL: http:// www.ars.usda.gov/SP2UserFiles/Place/80620510/ ALL\%20AVAIL\%20indices\%2016Jan014.pdf

Estrada, M.N., Vélez, P.E. 2003. Procedimientos para el registro, aislamiento, mantenimiento, preservaciónysistematización de una colección de hongos entomopatógenos. Manejo integrado de plagas y agroecología (Costa Rica) 70: 97103.

Fernandez Andreu, C.M., Martínez Machin, G., Perurena Lancha, M.R., Illnait Zaragozi, M.T., Valdés Hernández, I. 2005. La colección de cultivos de hongos del Instituto Pedro Kouri, funciones y retos. Revista Cubana de Medicina Tropical 57(3): 214-208.

Gutierrez, A.C., Tornesello-Galván, J., Manfrino, R.G., Hipperdinger, M., Falvo, M., D’Alessandro, C.P., López Lastra, C.C. 2017. Organización y conservación de la colección de hongos patógenos y simbiontes de insectos y otros artrópodos del CEPAVE (CONICET-UNLP), La Plata, Argentina. Revista argentina de Microbiología RAM 49(2): 183-188.

Humber, R.A. 2012. Identification of Entomopathogenic Fungi. pp. 151-187. En: Lacey, L. (Ed.). Manual of Techniques in Insect Pathology. 2da. Edition Academic Press. 484 pp.

Index Fungorum Partnership. 2012. Index Fungorum. CABI Bioscience, CBS-KNAW Fungal Diversity Centre and Landcare Research. URL: http://www.indexfungorum. org/names/Names.asp. Accedido: Noviembre, 2018.

López Lastra, C.C. 1988. Incidencia y acción patógena de hongos en insectos de la República Argentina. Tesis de Doctorado. Facultad de Ciencias Naturales, UNLP. 110 pp.

López Lastra, C.C., Gutierrez, A.C. 2019. Métodos de preservación de cultivos de hongos entomopatógenos. En: López Lastra, 
C.C., Lecuona, R.E. (Eds.) Micopatología de artrópodos: 167-178. Ediciones Inta, Buenos Aires.

Marcenaro Rodríguez, D. 2013. Catálogo de hongos en semillas de frijol (Phaseolus vulgaris L.) En: Nicaragua. Inta Nicaragua. 56 pp. URL: http://www.inta.gob.ni

Montes de Oca, N., González, R.A., Riverón, Y., Núñez, A., Villoch, A., Rodriguez, N. 2008. Establecimiento y desarrollo de la colección de cultivos del CENSA Revista de Salud Animal 30 (1): 17-24.

Montesinos-Matías, R., Ayala Zermeño, M.A., BerlangaPadilla, A.M. 2015. Manual para la conservación y mantenimiento de hongos entomopatógenos (Manual for the conservation and maintenance of entomopathogenic fungi). Servicio Nacional de Sanidad, Inocuidad y Calidad Agroalimentaria (SENASICA) Unidad de Promoción y Vinculación- SENASICA ISBN: 978-968-5384-08-7. México.
Montesinos-Matías, R., Ayala Zermeño, M.A., Berlanga-Padilla, A.M., Rodriguez Rodriguez, J.C. 2016. Colección de hongos entomopatógenos. Catálogo de especies. Sagarpa-Senasica México. 61 pp.

Panizo, M.M., Reviakina, V., Rodríguez-Lemoine, V., Dolande, M., Alarcón, V., Ferrara, G., García, N., Gonzalez, G. 2015. Micoteca del Instituto Nacional de Higiene Rafael Rangel: 60 años preservando la diversidad fúngica de interés médico en Venezuela. Revista de la Sociedad Venezolana de Microbiología 35: 4-12.

Sosa-Gómez, D.R., da Silva, J.J. 2002. Fungos entomopatogênicos, catálogo de isolados. Londrina, Embrapa Soja. 32 pp. URL: http://ainfo.cnptia.embrapa.br/digital/bitstream/ítem /60080/1/Documentos-188.pdf

Received: 21.01.2019

Accepted: 19.02.2020 